\title{
Flavor superconductivity from gauge/gravity duality
}

\author{
Martin Ammon, ${ }^{a}$ Johanna Erdmenger, ${ }^{a}$ Patrick Kerner ${ }^{a}$ and Matthias Kaminski ${ }^{b}$ \\ ${ }^{a}$ Max-Planck-Institut für Physik, Werner-Heisenberg-Institut, \\ Föhringer Ring 6, 80805 München, Germany \\ ${ }^{b}$ Instituto de Fisica Teórica UAM/CSIC Facultad de Ciencias, \\ C-XVI Universidad Autónoma de Madrid Cantoblanco, Madrid 28049, Spain \\ E-mail: ammon@mppmu.mpg.de, jke@mppmu.mpg.de, pkerner@mppmu.mpg.de, \\ matthias.kaminski@uam.es
}

ABSTRACT: We give a detailed account and extensions of a holographic flavor superconductivity model which we have proposed recently. The model has an explicit field theory realization as strongly coupled $\mathcal{N}=2$ Super Yang-Mills theory with fundamental matter at finite temperature and finite isospin chemical potential. Using gauge/gravity duality, i.e. a probe of two flavor D7-branes in the AdS black hole background, we show that the system undergoes a second order phase transition with critical exponent $1 / 2$. The new ground state may be interpreted as a $\rho$ meson superfluid. It shows signatures known from superconductivity, such as an infinite dc conductivity and a gap in the frequencydependent conductivity. We present a stringy picture of the condensation mechanism in terms of a recombination of strings. We give a detailed account of the evaluation of the non-Abelian Dirac-Born-Infeld action involved using two different methods. Finally we also consider the case of massive flavors and discuss the holographic Meissner-Ochsenfeld effect in our scenario.

KEYworDs: Gauge-gravity correspondence, D-branes, Black Holes

ARXIV EPRINT: 0903.1864 


\section{Contents}

$\begin{array}{llr}1 & \text { Introduction and summary } & 2\end{array}$

2 Field theory interpretation $\quad 4$

3 Holographic setup $\quad 6$

$\begin{array}{ll}3.1 & \text { Background and brane configuration }\end{array}$

$\begin{array}{lll}3.2 & \text { DBI action and equations of motion } & 7\end{array}$

3.2.1 Adapted symmetrized trace prescription 8

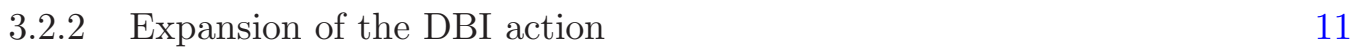

4 String theory picture $\quad 12$

5 Thermodynamics \& phase transition $\quad 14$

$\begin{array}{ll}5.1 \text { Adapted symmetrized trace prescription } & 15\end{array}$

$\begin{array}{ll}5.2 \text { Expansion of the DBI action } & 16\end{array}$

$\begin{array}{lll}5.3 & \text { Thermodynamical consistency } & 16\end{array}$

$\begin{array}{lll}5.4 & \text { Results \& comparison of the two prescriptions } & 17\end{array}$

6 Fluctuations $\quad 22$

6.1 Adapted symmetrized trace prescription 22

6.2 Expansion of the DBI action 24

6.3 Result \& comparison of the two prescriptions 24

$\begin{array}{lll}6.3 .1 & \text { Conductivity } & 24\end{array}$

$\begin{array}{ll}\text { 6.3.2 Spectral functions } & 26\end{array}$

$\begin{array}{lll}\text { 6.3.3 Comments on stability } & 28\end{array}$

$\begin{array}{lll}\text { 6.3.4 Dynamical mass generation } & 29\end{array}$

$\begin{array}{lll}7 & \text { Meissner-Ochsenfeld effect } & 30\end{array}$

8 Discussion and outlook 33

A Expansion of the DBI action to fourth order 34

$\begin{array}{ll}\text { B Fluctuations } & \mathbf{3 5}\end{array}$

B.1 Adapted symmetrized trace prescription $\quad 35$

B.2 Expansion of the DBI action to fourth order 36 


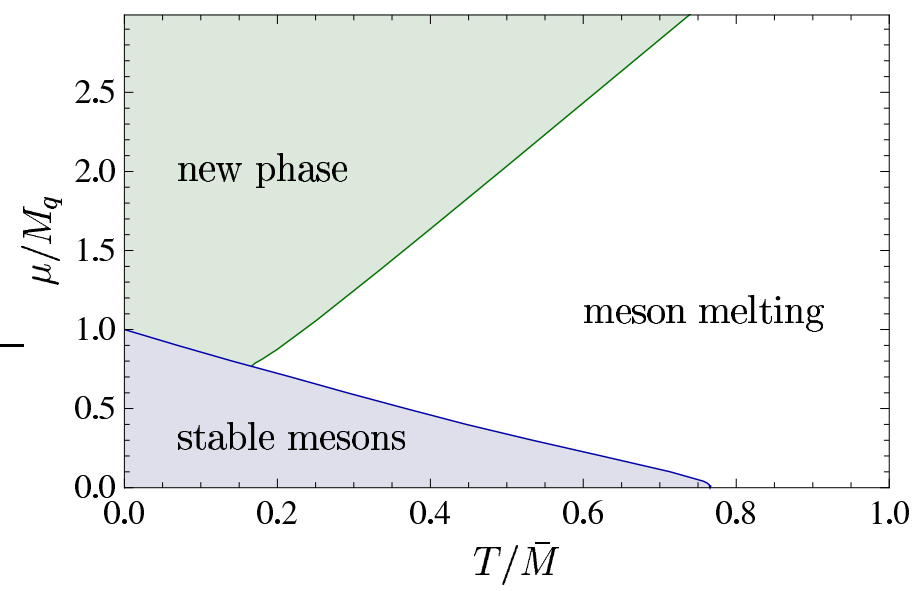

Figure 1. Phase diagram for fundamental matter in thermal strongly-coupled $\mathcal{N}=2 \mathrm{SYM}$ theory [32], with $\mu$ the isospin chemical potential, $M_{q}$ the bare quark mass, $\bar{M}=2 M_{q} \lambda^{-1 / 2}$, $\lambda$ the 't Hooft coupling and $T$ the temperature: In the blue shaded region, mesons are stable. In the white and green regions, the mesons melt. Here the new phase is stabilized while it was unstable in [32]. In this phase we find some features known from superconductivity.

\section{Introduction and summary}

Gauge/gravity duality has been used very successfully over the past years to obtain models with properties very similar to experimentally observed phenomena in both elementary and condensed matter physics. This is due to the fact that gauge/gravity duality is a powerful tool to compute observables of field theories at strong coupling [1]. In view of applications, it is particularly useful to turn on a temperature using an AdS black hole as gravity dual, as first proposed in [2]. In particular, gauge/gravity duality models which exhibit properties known from superconductivity have recently been constructed [3-22].

In this context we have recently proposed a model which represents a stringy realization of superconductivity in a relativistic framework [14]. The aim of the present paper is to give a detailed account of the setup and calculations involved in obtaining the results of [14], as well as further new results.

Our model is based on embedding a probe of two coincident D7 branes into the AdSblack hole background, which extends the original correspondence to a field theory with flavor degrees of freedom in the fundamental representation of the gauge group, along the lines of [23-25]. Using this approach, the correspondence has also been applied to finite flavor charge densities [26-32]. A finite charge density for the flavor symmetry is obtained by giving a vev to the time component of the gauge field on the D7 brane probe. In [32], a finite $\mathrm{SU}(2)$ isospin density $\tilde{d}$ was considered using a probe of two coincident D7 branes and switching on a gauge field $A=A_{0}^{3} \mathrm{~d} x_{0} \sigma^{3}$ on the probe. At high isospin densities $\tilde{d}>\tilde{d}_{c}$, this configuration becomes unstable against gauge field fluctuations $X=a^{1}+\mathrm{i} a^{2}$ and $Y=a^{1}-\mathrm{i} a^{2}$, corresponding to vector mesons [33] in the field theory (see figure 1) (see [34] for an early discussion of a similar instability). Note that we use capital letters for the background and small letters for the fluctuations. 
The central result obtained in this model [14] is that there is a solution to the equation of motion for the field on the two flavor branes, which in addition to the finite isospin density has a further vev of the form $A_{3}^{1} \mathrm{~d} x_{3} \sigma^{1}$. This solution has lower free energy in the new phase shown in figure 1 and stabilizes this phase. This applies at least to stability against the fluctuations $X$ and $Y$. Moreover we find that this phase has properties of a superconductor, such as a second order phase transition with a critical exponent of $1 / 2$. The flavor current, which is analogous to the electromagnetic current, displays infinite dc conductivity and a gap in the frequency-dependent conductivity. Furthermore, there is a dual string-theoretical picture of the Cooper pairs, as well as dynamical generation of meson masses.

The stabilization mechanism encountered in this context is related to the results of [35]. Within the Sakai-Sugimoto model, a vev of the form $A_{3}^{1} \mathrm{~d} x_{3} \sigma^{1}$ was considered in [36] in the context of $\rho$ meson condensation. To obtain a p-wave superconductor within a phenomenological AdS/QCD model, a vev of the form $A_{3}^{1} \mathrm{~d} x_{3} \sigma^{1}$ was first used in [7]. Note that in our model the equations of motion forbid a source term for $A_{3}^{1}$, i. e. the breaking of the $\mathrm{U}(1)$ is spontaneous.

The results presented here can be interpreted in two different ways. On the one hand, our results provide an important link between gauge/gravity with flavor and the recent gauge/gravity realizations of superconducting states e.g. in [3, 7, 13], as well as a realization of a superconducting current from a top-down (super-)gravity model, rather than in a phenomenological bottom-up AdS/QCD model. Moreover, the degrees of freedom in the dual field theory are identified explicitly.

On the other hand, our holographic description can also be considered as a realization of a meson superfluid. In the model presented here, the $\rho$ mesons form a superfluid. In QCD like theories, $\rho$ meson condensation is discussed in $[37,38]$. The corresponding picture is that the mesons move through the quark-gluon plasma without friction. Similarly, the absorption rate for radiation should be reduced for a specific frequency range [39]. For pions, similar results have been found within QCD: There are analytical results for small and very large isospin chemical potential using the chiral Lagrangian [40, 41], as well as lattice results [42]. Unlike the lattice results, the results presented here can easily be generalized to finite baryon chemical potential. A pion superfluid was obtained within gauge/gravity duality in [18].

Let us compare our results to QCD. In QCD, the pion condensate is of course the natural state in isospin asymmetric matter. The condensation of a particle sets in if the isospin chemical potential is larger than the mass of this particle. According to this rule, the pions condense first in QCD since they are the Nambu-Goldstone bosons of the spontaneous chiral symmetry breaking and therefore the lightest particles. However the dual field theory which we consider in this letter is supersymmetric at zero temperature and therefore chiral symmetry cannot be broken spontaneously. In this supersymmetric theory, the vector and scalar mesons have the same mass at zero temperature. Due to finite temperature effects, the mass of the vector and scalar mesons can become different as we increase the temperature. It is a priori unclear which particle will condense. In our model we checked that the vector mesons condense first such that the $\varrho$-meson condensation 
state, which we consider in this paper, is the physical ground state of our system near the phase transition.

In this paper we explain in detail the method used for evaluating the non-Abelian DiracBorn-Infeld action involved. Moreover we give a detailed account of our results for finite quark mass and the derivation of the Meissner effect. Results similar to ours have also been obtained in [15] shortly after [14]. However there the non-abelian DBI action is considered only to second order in $\alpha^{\prime}$. Some of the features discussed here are also present in [15]: a second order phase transition as well as a gap in the frequency-dependent conductivity. However, since higher order terms in $\alpha^{\prime}$ are not taken into account in [15], these authors are not able to see higher order excitations in the frequency-dependent conductivity. Here we identify these higher order peaks with meson resonances.

Moreover, we give a string-theoretical interpretation of the formation of a superconducting state of lower free energy: Essentially the condensation process corresponds to a recombination of strings. The detailed picture is as follows: A small isospin density corresponds to strings stretching from the D7 branes to the horizon [26], the horizon strings. These are located close to the horizon. Increasing the isospin density, the horizon strings strongly charge one of the D7 branes positively near the horizon, and the other negatively. At a critical value, it becomes energetically favorable for the horizon strings to recombine into D7-D7 strings which are free to move into the bulk and therefore lower the charge density near the horizon. The D7-D7 strings form the superconducting condensate. Thus they provide a picture of strongly coupled holographic Cooper pairs. Note that for the construction considered the non-Abelian structure generated by the two D7-branes is essential since it provides a charged condensate. The non-Abelian forces play an important role for stabilizing the condensate.

This paper is organized as follows: We begin with describing the field-theory action in section 2. We then explain the dual supergravity setup in section 3. In particular, we contrast and compare two different approaches to evaluating the non-Abelian DBI action involved. These are an adapted symmetrized trace prescription and the expansion of the DBI action to fourth order. We frequently refer to these approaches as prescriptions. We then explain the string-theoretical picture in detail in section 4 . Then we describe the thermodynamics of the phase transition in 5 , and in particular show that it is second order with critical exponent $1 / 2$. In 6 we evaluate the fluctuations, again in the two different approaches for the non-abelian DBI action. We calculate the frequency-dependent conductivity and show it displays the expected gap. Moreover we check that while the normal phase was unstable with respect to the fluctuations in $X$ and $Y$, the superconducting phase is now stable with respect to these fluctuations. Finally we show in 7 that our system displays a Meissner-Ochsenfeld effect. We conclude with a collection of remarks and suggestions in 8.

\section{Field theory interpretation}

In this paper we will focus on a $3+1-$ dimensional $\mathcal{N}=2$ supersymmetric $\mathrm{SU}\left(N_{c}\right)$ YangMills theory at temperature $T$, consisting of a $\mathcal{N}=4$ gauge multiplet as well as $N_{f}$ massive 
$\mathcal{N}=2$ supersymmetric hypermultiplets $\left(\psi_{i}, \phi_{i}\right)$. The hypermultiplets give rise to the flavor degrees transforming in the fundamental representation of the gauge group. The action is written down explicitly for instance in [43]. In particular, we work in the large $N_{c}$ limit with $N_{f} \ll N_{c}$ at strong coupling, i. e. with $\lambda \gg 1$, where $\lambda=g_{\mathrm{YM}}^{2} N_{c}$ is the 't Hooft coupling constant. In the following we will consider only two flavors, i. e. $N_{f}=2$. The flavor degrees of freedom are called $u$ and $d$. If the masses of the two flavor degrees are degenerate, the theory has a global $\mathrm{U}(2)$ flavor symmetry, whose overall $\mathrm{U}(1)_{B}$ subgroup can be identified with the baryon number.

In the following we will consider the theory at finite isospin chemical potential $\mu$, which is introduced as the source of the operator

$$
J_{0}^{3} \propto \bar{\psi} \sigma^{3} \gamma_{0} \psi+\phi \sigma^{3} \partial_{0} \phi=n_{u}-n_{d},
$$

where $n_{u / d}$ is the charge density of the isospin fields, $\left(\phi_{u}, \phi_{d}\right)=\phi$ and $\left(\psi_{u}, \psi_{d}\right)=\psi$. $\sigma^{i}$ are the Pauli matrices. A non-zero vev $\left\langle J_{0}^{3}\right\rangle$ introduces an isospin density as discussed in [32]. The isospin chemical potential $\mu$ explicitly breaks the $\mathrm{U}(2) \simeq \mathrm{U}(1)_{B} \times \mathrm{SU}(2)_{I}$ flavor symmetry down to $\mathrm{U}(1)_{B} \times \mathrm{U}(1)_{3}$, where $\mathrm{U}(1)_{3}$ is generated by the unbroken generator $\sigma^{3}$ of the $\mathrm{SU}(2)_{I}$. Under the $\mathrm{U}(1)_{3}$ symmetry the fields with index $u$ and $d$ have positive and negative charge, respectively.

However, the theory is unstable at large isospin chemical potential [32]. The new phase is sketched in figure 1. We show in this paper (see also [14]), that the new phase is stabilized by a non-vanishing vacuum expectation value of the current component

$$
J_{3}^{1} \propto \bar{\psi} \sigma^{1} \gamma_{3} \psi+\phi \sigma^{1} \partial_{3} \phi=\bar{\psi}_{u} \gamma_{3} \psi_{d}+\bar{\psi}_{d} \gamma_{3} \psi_{u}+\text { bosons } .
$$

This current component breaks both the $\mathrm{SO}(3)$ rotational symmetry as well as the remaining Abelian $\mathrm{U}(1)_{3}$ flavor symmetry spontaneously. The rotational $\mathrm{SO}(3)$ is broken down to $\mathrm{SO}(2)_{3}$, which is generated by rotations around the $x^{3}$ axis. Due to the non-vanishing vev for $J_{3}^{1}$, flavor charged vector mesons condense and form a superfluid. Let us emphasize that we do not describe a color superconductor on the field theory side, since the condensate is a gauge singlet.

Moreover, our results have a condensed matter interpretation: Our model can be considered as a holographic p-wave superconductor. The global $\mathrm{U}(1)_{3}$ in our model is the analog of the local U(1)em symmetry of electromagnetic interactions. So far in all holographic models of superconductors the breaking of a global symmetry on the field theory side is considered. In our model, the current $J^{3}$ corresponds to the electric current $J_{\mathrm{em}}$. The condensate $\left\langle J_{3}^{1}\right\rangle$ breaks the $\mathrm{U}(1)_{3}$ spontaneously. Therefore it can be viewed as the superconducting condensate, which is analogous to the Cooper pairs. Since the condensate $\left\langle J_{3}^{1}\right\rangle$ transforms as a vector under spatial rotations, it is a p-wave superconductor. - Strictly speaking, for a superconductor interpretation it would be necessary to gauge the global $\mathrm{U}(1)_{3}$ symmetry which is broken spontaneously in our model. A spontaneously broken global symmetry corresponds to a superfluid. However, many features of superconductivity do not depend on whether the $\mathrm{U}(1)_{3}$ is gauged. One exception to this is the Meissner-Ochsenfeld effect. To generate the currents expelling the magnetic field, the $\mathrm{U}(1)_{3}$ symmetry has to be gauged. This is discussed further below in section 7 . 


\section{$3 \quad$ Holographic setup}

\subsection{Background and brane configuration}

We consider asymptotically $A d S_{5} \times S^{5}$ space-time. The $A d S_{5} \times S^{5}$ geometry is holographically dual to the $\mathcal{N}=4$ Super Yang-Mills theory with gauge group $\mathrm{SU}\left(N_{c}\right)$. The dual description of a finite temperature field theory is an AdS black hole. We use the coordinates of [26] to write the AdS black hole background in Minkowski signature as

$$
d s^{2}=\frac{\varrho^{2}}{2 R^{2}}\left(-\frac{f^{2}}{\tilde{f}} \mathrm{~d} t^{2}+\tilde{f} \mathrm{~d} \vec{x}^{2}\right)+\left(\frac{R}{\varrho}\right)^{2}\left(\mathrm{~d} \varrho^{2}+\varrho^{2} \mathrm{~d} \Omega_{5}^{2}\right),
$$

with $\mathrm{d} \Omega_{5}^{2}$ the metric of the unit 5 -sphere and

$$
\begin{aligned}
& f(\varrho)=1-\frac{\varrho_{H}^{4}}{\varrho^{4}}, \\
& \tilde{f}(\varrho)=1+\frac{\varrho_{H}^{4}}{\varrho^{4}},
\end{aligned}
$$

where $R$ is the AdS radius, with

$$
R^{4}=4 \pi g_{s} N_{c} \alpha^{\prime 2}=2 \lambda \alpha^{\prime 2} .
$$

The temperature of the black hole given by (3.1) may be determined by demanding regularity of the Euclidean section. It is given by

$$
T=\frac{\varrho H}{\pi R^{2}} .
$$

In the following we may use the dimensionless coordinate $\rho=\varrho / \varrho_{H}$, which covers the range from the event horizon at $\rho=1$ to the boundary of the AdS space at $\rho \rightarrow \infty$.

To include fundamental matter, we embed $N_{f}$ coinciding D7-branes into the tendimensional space-time. These D7-branes host flavor gauge fields $A_{\mu}$ with gauge group $\mathrm{U}\left(N_{f}\right)$. To write down the DBI action for the D7-branes, we introduce spherical coordinates $\left\{r, \Omega_{3}\right\}$ in the 4567-directions and polar coordinates $\{L, \phi\}$ in the 89-directions [26]. The angle between these two spaces is denoted by $\theta(0 \leq \theta \leq \pi / 2)$. The six-dimensional space in the 456789-directions is given by

$$
\mathrm{d} \varrho^{2}+\varrho^{2} \mathrm{~d} \Omega_{5}^{2}=\mathrm{d} r^{2}+r^{2} \mathrm{~d} \Omega_{3}^{2}+\mathrm{d} L^{2}+L^{2} \mathrm{~d} \phi^{2}=\mathrm{d} \varrho^{2}+\varrho^{2}\left(\mathrm{~d} \theta^{2}+\cos ^{2} \theta \mathrm{d} \phi^{2}+\sin ^{2} \theta \mathrm{d} \Omega_{3}^{2}\right),
$$

where $r=\varrho \sin \theta, \varrho^{2}=r^{2}+L^{2}$ and $L=\varrho \cos \theta$. Due to the $\mathrm{SO}(4)$ rotational symmetry in the 4567 directions, the embedding of the D7-branes only depends on the radial coordinate $\rho$. Defining $\chi=\cos \theta$, we parametrize the embedding by $\chi=\chi(\rho)$ and choose $\phi=0$ using the $\mathrm{SO}(2)$ symmetry in the 89-direction. The induced metric $G$ on the D7-brane probes is then

$$
d s^{2}(G)=\frac{\varrho^{2}}{2 R^{2}}\left(-\frac{f^{2}}{\tilde{f}} \mathrm{~d} t^{2}+\tilde{f} \mathrm{~d} \vec{x}^{2}\right)+\frac{R^{2}}{\varrho^{2}} \frac{1-\chi^{2}+\varrho^{2}\left(\partial_{\varrho} \chi\right)^{2}}{1-\chi^{2}} \mathrm{~d} \varrho^{2}+R^{2}\left(1-\chi^{2}\right) \mathrm{d} \Omega_{3}^{2} .
$$


The square root of the determinant of $G$ is given by

$$
\sqrt{-G}=\frac{\sqrt{h_{3}}}{4} \varrho^{3} f \tilde{f}\left(1-\chi^{2}\right) \sqrt{1-\chi^{2}+\varrho^{2}\left(\partial_{\varrho} \chi\right)^{2}},
$$

where $h_{3}$ is the determinant of the 3 -sphere metric.

As in [32] we introduce a $\mathrm{SU}(2)$ isospin chemical potential $\mu$ by a non-vanishing time component of the non-Abelian background field on the D7-brane. The generators of the $\mathrm{SU}(2)$ gauge group are given by the Pauli matrices $\sigma^{i}$. Due to the gauge symmetry, we may rotate the flavor coordinates until the chemical potential lies in the third flavor direction,

$$
\mu=\lim _{\rho \rightarrow \infty} A_{0}^{3}(\rho)
$$

This non-zero gauge field breaks the $\mathrm{SU}(2)$ gauge symmetry down to $\mathrm{U}(1)_{3}$ generated by the third Pauli matrix $\sigma^{3}$. The spacetime symmetry on the boundary is still $\mathrm{SO}(3)$. Notice that the Lorentz group $\mathrm{SO}(3,1)$ is already broken down to $\mathrm{SO}(3)$ by the finite temperature. In addition, we consider a further non-vanishing background gauge field which stabilizes the system for large chemical potentials. Due to the symmetry of our setup we may choose $A_{3}^{1} \mathrm{~d} x^{3} \sigma^{1}$ to be non-zero. To obtain an isotropic configuration in the field theory, this new gauge field $A_{3}^{1}$ only depends on $\rho$. Due to this two non-vanishing gauge fields, the field strength tensor on the branes has the following non-zero components,

$$
\begin{aligned}
& F_{\varrho 3}^{1}=-F_{3 \varrho}^{1}=\partial_{\varrho} A_{3}^{1}, \\
& F_{03}^{2}=-F_{30}^{2}=\frac{\gamma}{\sqrt{\lambda}} A_{0}^{3} A_{3}^{1}, \\
& F_{\varrho 0}^{3}=-F_{0 \varrho}^{3}=\partial_{\varrho} A_{0}^{3} .
\end{aligned}
$$

\subsection{DBI action and equations of motion}

In this section we calculate the equations of motion which determine the profile of the D7-brane probes and of the gauge fields on these branes. An discussion of the gauge field profiles, which we use to give a geometrical interpretation of the stabilization of the system and the pairing mechanism, may be found in section 4 .

The DBI action determines the shape of the brane embeddings, i. e. the scalar fields $\phi$, as well as the configuration of the gauge fields $A$ on these branes. We consider the case of $N_{f}=2$ coincident D7-branes for which the non-Abelian DBI action reads [44]

$$
S_{\mathrm{DBI}}=-T_{D 7} \operatorname{Str} \int \mathrm{d}^{8} \xi \sqrt{\operatorname{det} Q}\left[\operatorname{det}\left(P_{a b}\left[E_{\mu \nu}+E_{\mu i}\left(Q^{-1}-\delta\right)^{i j} E_{j \nu}\right]+2 \pi \alpha^{\prime} F_{a b}\right)\right]^{\frac{1}{2}}
$$

with

$$
Q^{i}{ }_{j}=\delta^{i}{ }_{j}+\mathrm{i} 2 \pi \alpha^{\prime}\left[\Phi^{i}, \Phi^{k}\right] E_{k j}
$$

and $P_{a b}$ the pullback to the $\mathrm{D} p$-brane, where for a $\mathrm{D} p$-brane in $d$ dimensions we have $\mu, \nu=0, \ldots,(d-1), a, b=0, \ldots, p, i, j=(p+1), \ldots,(d-1), E_{\mu \nu}=g_{\mu \nu}+B_{\mu \nu}$. In our case we set $p=7, d=10, B \equiv 0$. As in [32] we can simplify this action significantly by 
using the spatial and gauge symmetries present in our setup. The action becomes

$$
\begin{aligned}
S_{\mathrm{DBI}}=-T_{D 7} \int \mathrm{d}^{8} \xi \operatorname{Str} \sqrt{\left|\operatorname{det}\left(G+2 \pi \alpha^{\prime} F\right)\right|} & \\
=-T_{D 7} \int \mathrm{d}^{8} \xi \sqrt{-G} \operatorname{Str}\left[1+G^{00} G^{44}\left(F_{\varrho 0}^{3}\right)^{2}\left(\sigma^{3}\right)^{2}+\right. & G^{33} G^{44}\left(F_{\varrho 3}^{1}\right)^{2}\left(\sigma^{1}\right)^{2} \\
& \left.+G^{00} G^{33}\left(F_{03}^{2}\right)^{2}\left(\sigma^{2}\right)^{2}\right]^{\frac{1}{2}},
\end{aligned}
$$

where in the second line the determinant is calculated. Due to the symmetric trace, all commutators between the matrices $\sigma^{i}$ vanish. It is known that the symmetrized trace prescription in the DBI action is only valid up to fourth order in $\alpha^{\prime}[45,46]$. However the corrections to the higher order terms are suppressed by $N_{f}^{-1}$ [47] (see also [48]). Here we use two different approaches to evaluate the non-Abelian DBI action (3.12). First, we modify the symmetrized trace prescription by omitting the commutators of the generators $\sigma^{i}$ and then setting $\left(\sigma^{i}\right)^{2}=1$ (see subsection 3.2.1 below). This prescription makes the calculation of the full DBI action feasible. Second, we expand the non-Abelian DBI action to fourth order in the field strength $F$ (see subsection 3.2.2).

\subsubsection{Adapted symmetrized trace prescription}

Using the adapted symmetrized trace prescription defined above, the action becomes

$$
\begin{aligned}
S_{\mathrm{DBI}} & =-T_{D 7} N_{f} \int \mathrm{d}^{8} \xi \sqrt{-G}\left[1+G^{00} G^{44}\left(F_{\varrho}^{3}\right)^{2}+G^{33} G^{44}\left(F_{\varrho 3}^{1}\right)^{2}+G^{00} G^{33}\left(F_{03}^{2}\right)^{2}\right]^{\frac{1}{2}} \\
& =-\frac{T_{D 7} N_{f}}{4} \int \mathrm{d}^{8} \xi \varrho^{3} f \tilde{f}\left(1-\chi^{2}\right) \Upsilon(\rho, \chi, \tilde{A}),
\end{aligned}
$$

with

$$
\begin{aligned}
\Upsilon(\rho, \chi, \tilde{A})=\left[1-\chi^{2}+\rho^{2}\left(\partial_{\rho} \chi\right)^{2}-\frac{2 \tilde{f}}{f^{2}}\left(1-\chi^{2}\right)\left(\partial_{\rho} \tilde{A}_{0}^{3}\right)^{2}+\frac{2}{\tilde{f}}\left(1-\chi^{2}\right)\left(\partial_{\rho} \tilde{A}_{3}^{1}\right)^{2}\right. \\
\left.-\frac{2 \gamma^{2}}{\pi^{2} \rho^{4} f^{2}}\left(1-\chi^{2}+\rho^{2}\left(\partial_{\rho} \chi\right)^{2}\right)\left(\tilde{A}_{0}^{3} \tilde{A}_{3}^{1}\right)^{2}\right]^{\frac{1}{2}},
\end{aligned}
$$

where the dimensionless quantities $\rho=\varrho / \varrho_{H}$ and $\tilde{A}=\left(2 \pi \alpha^{\prime}\right) A / \varrho_{H}$ are used. To obtain first order equations of motion for the gauge fields which are easier to solve numerically, we perform a Legendre transformation. Similarly to $[26,32]$ we calculate the electric displacement $p_{0}^{3}$ and the magnetizing field $p_{3}^{1}$ which are given by the conjugate momenta of the gauge fields $A_{0}^{3}$ and $A_{3}^{1}$,

$$
p_{0}^{3}=\frac{\delta S_{\mathrm{DBI}}}{\delta\left(\partial_{\varrho} A_{0}^{3}\right)}, \quad p_{3}^{1}=\frac{\delta S_{\mathrm{DBI}}}{\delta\left(\partial_{\varrho} A_{3}^{1}\right)} .
$$

In contrast to $[26,27,29,32]$, the conjugate momenta are not constant any more but depend on the radial coordinate $\varrho$ due to the non-Abelian term $A_{0}^{3} A_{3}^{1}$ in the DBI action. For the dimensionless momenta $\tilde{p}_{0}^{3}$ and $\tilde{p}_{3}^{1}$ defined as

$$
\tilde{p}=\frac{p}{2 \pi \alpha^{\prime} N_{f} T_{D 7} \varrho_{H}^{3}},
$$


we get

$$
\tilde{p}_{0}^{3}=\frac{\rho^{3} \tilde{f}^{2}\left(1-\chi^{2}\right)^{2} \partial_{\rho} \tilde{A}_{0}^{3}}{2 f \Upsilon(\rho, \chi, \tilde{A})}, \quad \tilde{p}_{3}^{1}=-\frac{\rho^{3} f\left(1-\chi^{2}\right)^{2} \partial_{\rho} \tilde{A}_{3}^{1}}{2 \Upsilon(\rho, \chi, \tilde{A}))}
$$

Finally, the Legendre-transformed action is given by

$$
\begin{aligned}
\tilde{S}_{\mathrm{DBI}} & =S_{\mathrm{DBI}}-\int \mathrm{d}^{8} \xi\left[\left(\partial_{\varrho} A_{0}^{3}\right) \frac{\delta S_{\mathrm{DBI}}}{\delta\left(\partial_{\varrho} A_{0}^{3}\right)}+\left(\partial_{\varrho} A_{3}^{1}\right) \frac{\delta S_{\mathrm{DBI}}}{\delta\left(\partial_{\varrho} A_{3}^{1}\right)}\right] \\
& =-\frac{T_{D 7} N_{f}}{4} \int \mathrm{d}^{8} \xi \varrho^{3} f \tilde{f}\left(1-\chi^{2}\right) \sqrt{1-\chi^{2}+\rho^{2}\left(\partial_{\rho} \chi\right)^{2}} V(\rho, \chi, \tilde{A}, \tilde{p}),
\end{aligned}
$$

with

$$
V(\rho, \chi, \tilde{A}, \tilde{p})=\left[\left(1-\frac{2 \gamma^{2}}{\pi^{2} \rho^{4} f^{2}}\left(\tilde{A}_{0}^{3} \tilde{A}_{3}^{1}\right)^{2}\right)\left(1+\frac{8\left(\tilde{p}_{0}^{3}\right)^{2}}{\rho^{6} \tilde{f}^{3}\left(1-\chi^{2}\right)^{3}}-\frac{8\left(\tilde{p}_{3}^{1}\right)^{2}}{\rho^{6} \tilde{f} f^{2}\left(1-\chi^{2}\right)^{3}}\right)\right]^{\frac{1}{2}} .
$$

Then the first order equations of motion for the gauge fields and their conjugate momenta are

$$
\begin{aligned}
\partial_{\rho} \tilde{A}_{0}^{3} & =\frac{2 f \sqrt{1-\chi^{2}+\rho^{2}\left(\partial_{\rho} \chi\right)^{2}}}{\rho^{3} \tilde{f}^{2}\left(1-\chi^{2}\right)^{2}} \tilde{p}_{0}^{3} W(\rho, \chi, \tilde{A}, \tilde{p}), \\
\partial_{\rho} \tilde{A}_{3}^{1} & =-\frac{2 \sqrt{1-\chi^{2}+\rho^{2}\left(\partial_{\rho} \chi\right)^{2}}}{\rho^{3} f\left(1-\chi^{2}\right)^{2}} \tilde{p}_{3}^{1} W(\rho, \chi, \tilde{A}, \tilde{p}), \\
\partial_{\rho} \tilde{p}_{0}^{3} & =\frac{\tilde{f}\left(1-\chi^{2}\right) \sqrt{1-\chi^{2}+\rho^{2}\left(\partial_{\rho} \chi\right)^{2}} c^{2}}{2 \pi^{2} \rho f W(\rho, \chi, \tilde{A}, \tilde{p})}\left(\tilde{A}_{3}^{1}\right)^{2} \tilde{A}_{0}^{3}, \\
\partial_{\rho} \tilde{p}_{3}^{1} & =\frac{\tilde{f}\left(1-\chi^{2}\right) \sqrt{1-\chi^{2}+\rho^{2}\left(\partial_{\rho} \chi\right)^{2}} c^{2}}{2 \pi^{2} \rho f W(\rho, \chi, \tilde{A}, \tilde{p})}\left(\tilde{A}_{0}^{3}\right)^{2} \tilde{A}_{3}^{1},
\end{aligned}
$$

with

$$
W(\rho, \chi, \tilde{A}, \tilde{p})=\sqrt{\frac{1-\frac{2 \gamma^{2}}{\pi^{2} \rho^{4} f^{2}}\left(\tilde{A}_{0}^{3} \tilde{A}_{3}^{1}\right)^{2}}{1+\frac{8\left(\tilde{p}_{0}^{3}\right)^{2}}{\rho^{6} \tilde{f}^{3}\left(1-\chi^{2}\right)^{3}}-\frac{8\left(\tilde{p}_{3}^{1}\right)^{2}}{\rho^{6} \tilde{f} f^{2}\left(1-\chi^{2}\right)^{3}}}} .
$$

For the embedding function $\chi$ we get the second order equation of motion

$$
\begin{aligned}
\partial_{\rho}\left[\frac{\rho^{5} f \tilde{f}\left(1-\chi^{2}\right)\left(\partial_{\rho} \chi\right)}{\sqrt{1-\chi^{2}+\rho^{2}\left(\partial_{\rho} \chi\right)^{2}}} V\right]=- & \frac{\rho^{3} f \tilde{f} \chi}{\sqrt{1-\chi^{2}+\rho^{2}\left(\partial_{\rho} \chi\right)^{2}}}\left[\left[3\left(1-\chi^{2}\right)+2 \rho^{2}\left(\partial_{\rho} \chi\right)^{2}\right] V\right. \\
& \left.-\frac{24\left(1-\chi^{2}+\rho^{2}\left(\partial_{\rho} \chi\right)^{2}\right)}{\tilde{f}^{3} \rho^{6}\left(1-\chi^{2}\right)^{3}} W\left(\left(\tilde{p}_{0}^{3}\right)^{2}-\frac{\tilde{f}^{2}}{f^{2}}\left(\tilde{p}_{3}^{1}\right)\right)\right] .
\end{aligned}
$$

We solve the equations numerically and determine the solution by integrating the equations of motion from the horizon at $\rho=1$ to the boundary at $\rho=\infty$. The initial conditions may 
be determined by the asymptotic expansion of the gravity fields near the horizon

$$
\begin{array}{lrl}
\tilde{A}_{0}^{3}= & \frac{c_{0}}{\sqrt{\left(1-\chi_{0}^{2}\right)^{3}+c_{0}^{2}}}(\rho-1)^{2} & +\mathcal{O}\left((\rho-1)^{3}\right) \\
\tilde{A}_{3}^{1}=b_{0} & +\mathcal{O}\left((\rho-1)^{3}\right) \\
\tilde{p}_{0}^{3}=c_{0} & +\frac{\gamma^{2} b_{0}^{2} c_{0}}{8 \pi^{2}}(\rho-1)^{2}+\mathcal{O}\left((\rho-1)^{3}\right) \\
\tilde{p}_{3}^{1}= & +\mathcal{O}\left((\rho-1)^{3}\right) \\
\chi=\chi_{0} & -\frac{3 \chi_{0}\left(1-\chi_{0}^{2}\right)^{3}}{4\left[\left(1-\chi_{0}^{2}\right)^{3}+c_{0}^{2}\right]}(\rho-1)^{2} & +\mathcal{O}\left((\rho-1)^{3}\right)
\end{array}
$$

where the terms in the expansions are arranged according to their order in $\rho-1$. For the numerical calculation we consider the terms up to sixth order in $\rho-1$. The three independent parameter $b_{0}, c_{0}$ and $\chi_{0}$ may be determined by field theory quantities defined via the asymptotic expansion of the gravity fields near the boundary,

$$
\begin{array}{llll}
\tilde{A}_{0}^{3}=\tilde{\mu} & -\frac{\tilde{d}_{0}^{3}}{\rho^{2}} & +\mathcal{O}\left(\rho^{-4}\right), \\
\tilde{A}_{3}^{1}= & -\frac{\tilde{d}_{3}^{1}}{\rho^{2}} & +\mathcal{O}\left(\rho^{-4}\right), \\
\tilde{p}_{0}^{3}=\tilde{d}_{0}^{3} & & +\mathcal{O}\left(\rho^{-4}\right) \\
\tilde{p}_{3}^{1}=-\tilde{d}_{3}^{1} & +\frac{\gamma^{2} \tilde{\mu}^{2} \tilde{d}_{3}^{1}}{4 \pi^{2} \rho^{2}} & +\mathcal{O}\left(\rho^{-4}\right) \\
\chi= & \frac{m}{\rho} & +\frac{c}{\rho^{3}}+\mathcal{O}\left(\rho^{-4}\right)
\end{array} .
$$

According to the AdS/CFT dictionary, $\mu$ is the isospin chemical potential. The parameters $\tilde{d}$ are related to the vev of the flavor currents $J$ by

$$
\tilde{d}_{0}^{3}=\frac{2^{\frac{5}{2}}\left\langle J_{0}^{3}\right\rangle}{N_{f} N_{c} \sqrt{\lambda} T^{3}}, \quad \tilde{d}_{3}^{1}=\frac{2^{\frac{5}{2}}\left\langle J_{3}^{1}\right\rangle}{N_{f} N_{c} \sqrt{\lambda} T^{3}}
$$

and $m$ and $c$ to the bare quark mass $M_{q}$ and the quark condensate $\langle\bar{\psi} \psi\rangle$,

$$
m=\frac{2 M_{q}}{\sqrt{\lambda} T}, \quad c=-\frac{8\langle\bar{\psi} \psi\rangle}{\sqrt{\lambda} N_{f} N_{c} T^{3}},
$$

respectively. There are two independent physical parameters, e.g. $m$ and $\mu$, in the grand canonical ensemble. From the boundary asymptotics (3.24), we also obtain that there is no source term for the current $J_{3}^{1}$. Therefore as a non-trivial result we find that the $\mathrm{U}(1)_{3}$ symmetry is always broken spontaneously. In contrast, in the related works on p-wave superconductors in $2+1$ dimensions $[7,8]$, the spontaneous breaking of the $\mathrm{U}(1)_{3}$ symmetry has to be put in by hand by setting the source term for the corresponding operator to zero. With the constraint $\left.\tilde{A}_{3}^{1}\right|_{\rho \rightarrow \infty}=0$ and the two independent physical parameters, we may fix the three independent parameters of the near-horizon asymptotics and obtain a solution to the equations of motion. 


\subsubsection{Expansion of the DBI action}

We now outline the second approach which we use. Expanding the action (3.12) to fourth order in the field strength $F$ yields

$$
S_{\mathrm{DBI}}=-T_{D 7} N_{f} \int \mathrm{d}^{8} \xi \sqrt{-G}\left[1+\frac{\mathcal{T}_{2}}{2}-\frac{\mathcal{T}_{4}}{8}+\cdots\right]
$$

where $\mathcal{T}_{i}$ consists of the terms with order $i$ in $F$. To calculate the $\mathcal{T}_{i}$, we use the following results for the symmetrized traces

$$
\begin{array}{ll}
2 \sigma: & \operatorname{Str}\left[\left(\sigma^{i}\right)^{2}\right]=N_{f}, \\
4 \sigma: & \operatorname{Str}\left[\left(\sigma^{i}\right)^{4}\right]=N_{f}, \quad \operatorname{Str}\left[\left(\sigma^{i}\right)^{2}\left(\sigma^{j}\right)^{2}\right]=\frac{N_{f}}{3},
\end{array}
$$

where the indices $i, j$ are distinct. Notice that the symmetric trace of terms with unpaired $\sigma$ matrices vanish, e. g. $\operatorname{Str}\left[\sigma^{i} \sigma^{j}\right]=N_{f} \delta^{i j}$. The $\mathcal{T}_{i}$ are given in the appendix A.

To perform the Legendre transformation of the above action, we determine the conjugate momenta as in (3.15). However, we cannot easily solve these equations for the derivative of the gauge fields since we obtain two coupled equations of third degree. Thus we directly calculate the equations of motion for the gauge fields on the D7-branes. The equations are given in (A.3) of the appendix.

To solve these equations, we use the same strategy as in the adapted symmetrized trace prescription discussed above. We integrate the equations of motion from the horizon at $\rho=1$ to the boundary at $\rho=\infty$ numerically. The initial conditions may be determined by the asymptotic behavior of the gravity fields near the horizon

$$
\begin{array}{ll}
\tilde{A}_{0}^{3}=\quad a_{2}(\rho-1)^{2} & +\mathcal{O}\left((\rho-1)^{3}\right), \\
\tilde{A}_{3}^{1}=b_{0} & +\mathcal{O}\left((\rho-1)^{3}\right), \\
\chi=\chi_{0}+\frac{3\left(a_{2}^{4}+4 a_{2}^{2}-8\right) \chi_{0}}{4\left(3 a_{2}^{4}+4 a_{2}^{2}+8\right)}(\rho-1)^{2} & +\mathcal{O}\left((\rho-1)^{3}\right) .
\end{array}
$$

For the numerical calculation we use the asymptotic expansion up to sixth order. As in the adapted symmetrized trace prescription, there are again three independent parameters $a_{2}, b_{0}, \chi_{0}$. Since we have not performed a Legendre transformation, we trade the independent parameter $c_{0}$ in the asymptotics of the conjugate momenta $\tilde{p}_{0}^{3}$ in the symmetrized trace prescription with the independent parameter $a_{2}$ (cf. asymptotics in equation (3.23)). However, the three independent parameters may again be determined in field theory quantities which are defined by the asymptotics of the gravity fields near the boundary

$$
\begin{array}{llll}
\tilde{A}_{0}^{3}=\mu & -\frac{\tilde{d}_{0}^{3}}{\rho^{2}} & +\mathcal{O}\left(\rho^{4}\right), \\
\tilde{A}_{3}^{1}= & -\frac{\tilde{d}_{3}^{1}}{\rho^{2}} & +\mathcal{O}\left(\rho^{4}\right), \\
\chi= & \frac{m}{\rho} & +\frac{c}{\rho^{3}} & +\mathcal{O}\left(\rho^{4}\right) .
\end{array}
$$




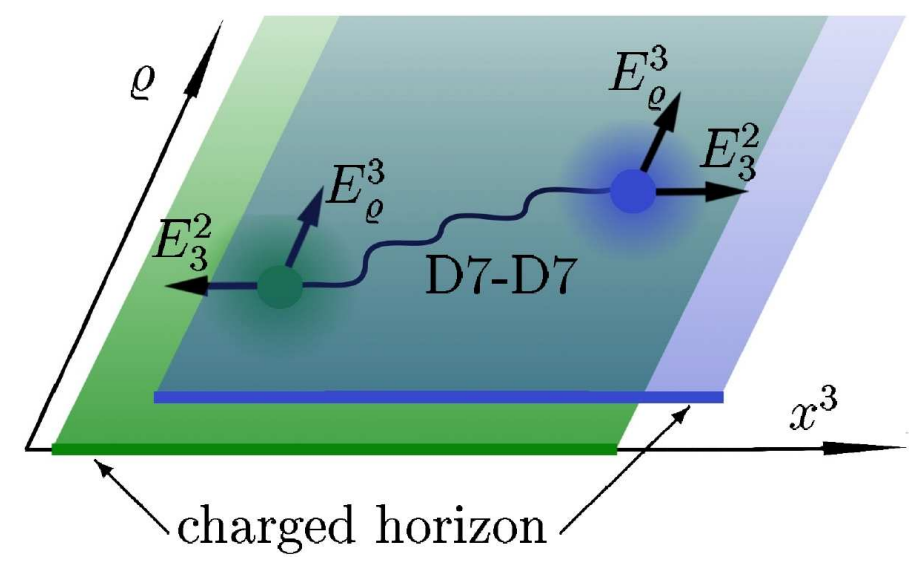

Figure 2. Sketch of our string setup: The figure shows the two coincident D7 branes stretched from the black hole horizon to the boundary as a green and a blue plane, respectively. Strings spanned from the horizon of the AdS black hole to the D7-branes induce a charge at the horizon [26, 27, 32]. However, above a critical charge density, the strings charging the horizon recombine to D7-D7 strings. These D7-D7 strings are shown in the figure. Whereas the fundamental strings stretched between the horizon and the D7-brane are localized near the horizon, the D7-D7 strings propagate into the bulk balancing the flavorelectric and gravitational, i.e. tension forces (see text). Thus these D7-D7 strings distribute the isospin charges along the AdS radial coordinate, leading to a stable configuration of reduced energy. This configuration of D7-D7 strings corresponds to a superconducting condensate.

The independent parameters $\mu, \tilde{d}_{0}^{3}, \tilde{d}_{3}^{1}, m, c$ are given by field theory quantities as presented in (3.25) and (3.26). Again we find that there is no source term for the current $J_{3}^{1}$, which implies spontaneous symmetry breaking. Therefore the independent parameters in both prescriptions are the same and we can use the same strategy to solve the equations of motion as described below (3.26).

\section{String theory picture}

In this section we give a string theory interpretation, i. e. a geometrical picture, of the formation of a new phase, for which the field theory is discussed in section 2. We show that the system is stabilized by dynamically generating a non-zero vev of the current component $J_{3}^{1}$ dual to the gauge field $A_{3}^{1}$ on the brane. Moreover, we find a geometrical picture of the pairing mechanism which forms the condensate $\left\langle J_{3}^{1}\right\rangle$, the Cooper pairs.

Let us first describe the unstable configuration in absence of the field $A_{3}^{1}$. As known from $[26,27,32]$, the non-zero field $A_{0}^{3}$ is induced by fundamental strings which are stretched from the D7-brane to the horizon of the black hole. In the subsequent we call these strings 'horizon strings'. Since the tension of these strings would increase as they move to the boundary, they are localized at the horizon, i. e. the horizon is effectively charged under the isospin charge given by (2.1). By increasing the horizon string density, the isospin charge on the D7-brane at the horizon and therefore the energy of the system grows. In [32], the critical density was found beyond which this setup becomes unstable. In this 
case, the strings would prefer to move towards the boundary due to the repulsive force on their charged endpoints generated by the flavorelectric field $E_{\varrho}^{3}=F_{0 \varrho}^{3}=-\partial_{\varrho} A_{0}^{3}$.

The setup is now stabilized by the new non-zero field $A_{3}^{1}$. This field is induced by D7D7 strings moving in the $x^{3}$ direction. This movement of the strings may be interpreted as a current in $x^{3}$ direction which induces the magnetic field $B_{3 \varrho}^{1}=F_{3 \varrho}^{1}=-\partial_{\varrho} A_{3}^{1}$. Moreover, the non-Abelian interaction between the D7-D7 strings and the horizon strings induces an flavorelectric field $E_{3}^{2}=F_{30}^{2}=\gamma / \sqrt{\lambda} A_{0}^{3} A_{3}^{1}$.

From the profile of the gauge fields and their conjugate momenta (see figure 3 ) we obtain the following: For $A_{3}^{1}=0$, i. e. in the normal phase $\left(T \geq T_{c}\right)$, the isospin density $\tilde{d}_{0}^{3}$ is exclusively generated at the horizon by the horizon strings. This can also be understood by the profile of the conjugate momenta $p_{0}^{3}$ (see figure $3(\mathrm{c})$ ). We interpret $p_{0}^{3}\left(\rho^{*}\right)$ as the isospin charge located between the horizon at $\rho=1$ and a fictitious boundary at $\rho=\rho^{*}$. In the normal phase, the momentum $p_{0}^{3}$ is constant along the radial direction $\rho$ (see figure $3(\mathrm{c}$ ), blue curve), and therefore the isospin density is exclusively generated at the horizon. In the superconducting phase where $A_{3}^{1} \neq 0$, i. e. $T<T_{c}$, the momentum $p_{0}^{3}$ is not constant any more. Its value increases monotonically towards the boundary and asymptotes to $\tilde{d}_{0}^{3}$ (see figure 3(c), red curve). Thus the isospin charge is also generated in the bulk and not only at the horizon. This decreases the isospin charge at the horizon and stabilizes the system.

Now we describe the string dynamics which distributes the isospin charge into the bulk. Since the field $A_{3}^{1}$ induced by the D7-D7 strings is non-zero in the superconducting phase, these strings must be responsible for stabilizing this phase. In the normal phase, there are only horizon strings. In the superconducting phase, some of these strings recombine to form D7-D7 strings which correspond to the non-zero gauge field $A_{3}^{1}$ and carry isospin charge. ${ }^{1}$ There are two forces acting on the D7-D7 strings, the flavorelectric force induced by the field $E_{\varrho}^{3}$ and the gravitational force between the strings and the black hole. The flavorelectric force points to the boundary while the gravitational force points to the horizon. The gravitational force is determined by the change in effective string tension, which contains the $\varrho$ dependent warp factor. The position of the D7-D7 strings is determined by the equilibrium of these two forces. Therefore the D7-D7 strings propagate from the horizon into the bulk and distribute the isospin charge.

Since the D7-D7 strings induce the field $A_{3}^{1}$, they also generate the density $\tilde{d}_{3}^{1}$ dual to the condensate $\left\langle J_{3}^{1}\right\rangle$, the Cooper pairs. This density $\tilde{d}_{3}^{1}$ is proportional to the D7-D7 strings located in the bulk, in the same way as the density $\tilde{d}_{0}^{3}$ counts the strings which carry isospin charge [26]. This suggest that we can also interpret $p_{3}^{1}\left(\rho^{*}\right)$ as the number of D7-D7 strings which are located between the horizon at $\rho=1$ and the fictitious boundary at $\rho=\rho^{*}$. The momentum $p_{3}^{1}$ is always zero at the horizon and increases monotonically in the bulk (see figure $3(\mathrm{~d})$ ). Thus there are no D7-D7 strings at the horizon.

The double importance of the D7-D7 strings is given by the fact that they are both responsible for stabilizing the superconducting phase by lowering the isospin charge density, as well as being the dual of the Cooper pairs since they break the $\mathrm{U}(1)_{3}$ symmetry.

\footnotetext{
${ }^{1}$ Note that the D7-D7 strings are of the same order as the horizon strings, namely $N_{f} / N_{c}$, since they originate from the DBI action [27].
} 


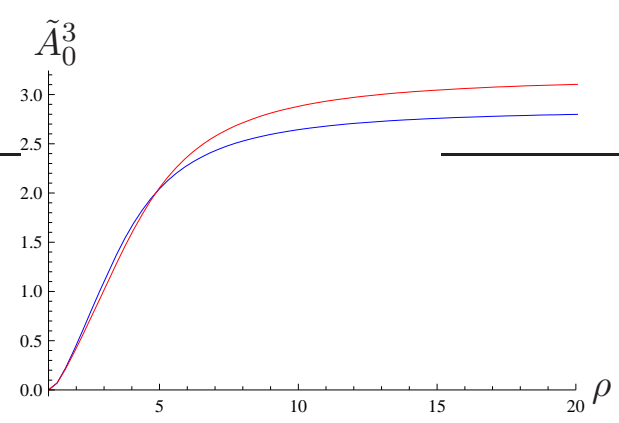

(a)

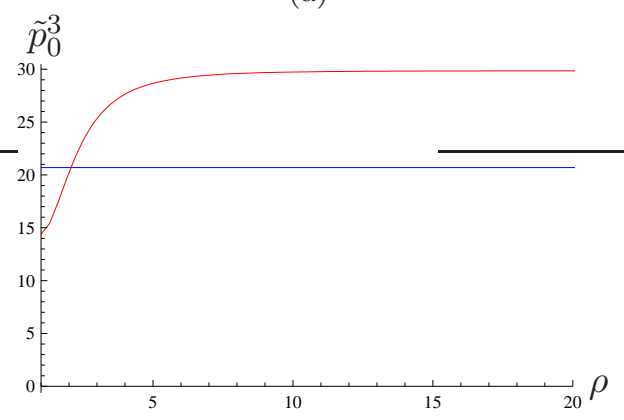

(c)

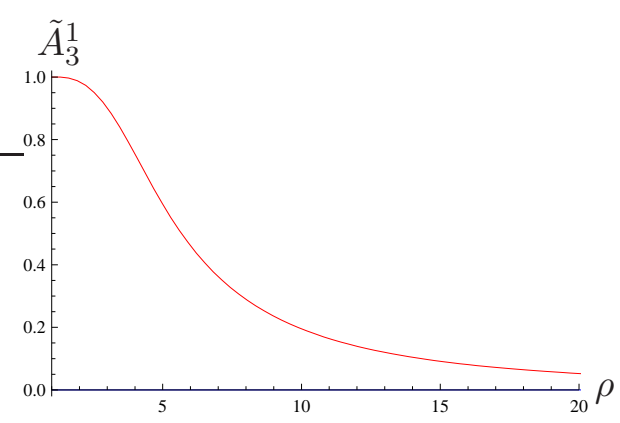

(b)

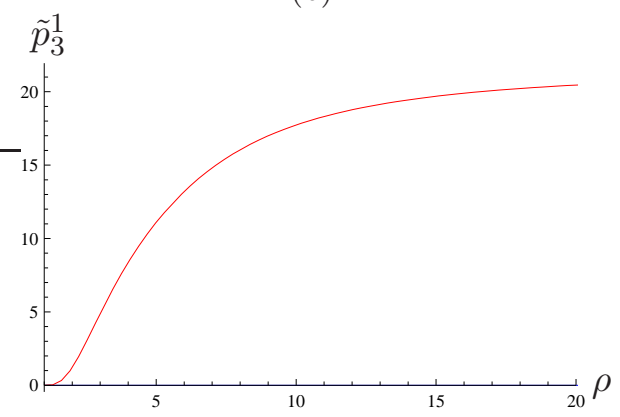

(d)

Figure 3. Profiles of the relevant dimensionless gauge fields $\tilde{A}$ on the D7-branes and their dimensionless conjugate momenta $\tilde{p}$ versus the dimensionless AdS radial coordinate $\rho$ near the horizon at $\rho=1$. The different curves correspond to the temperature $T=T_{c}$ (blue) and $T \approx 0.9 T_{c}$ (red). The plots are obtained at zero quark mass $m=0$ and by using the adapted symmetrized trace prescription. Similar plots may also be obtained at finite mass $m \neq 0$ and by using the DBI action expanded to fourth order in $F$. These plots show the same features: (a) The gauge field $\tilde{A}_{0}^{3}$ increases monotonically towards the boundary. At the boundary, its value is given by the dimensionless chemical potential $\tilde{\mu}$. (b) The gauge field $\tilde{A}_{3}^{1}$ is zero for $T \geq T_{c}$. For $T<T_{c}$, its value is non-zero at the horizon and decreases monotonically towards the boundary where its value has to be zero. (c) The conjugate momentum $\tilde{p}_{0}^{3}$ of the gauge field $\tilde{A}_{0}^{3}$ is constant for $T \geq T_{c}$. For $T<T_{c}$, its value increases monotonically towards the boundary. Its boundary value is given by the dimensionless density $\tilde{d}_{0}^{3}$. (d) The conjugate momentum $\tilde{p}_{3}^{1}$ of the gauge field $\tilde{A}_{3}^{1}$ is zero for $T \geq T_{c}$. For $T<T_{c}$, its value increases monotonically towards the boundary. Its boundary value is given by the dimensionless density $-\tilde{d}_{3}^{1}$.

\section{Thermodynamics \& phase transition}

In this section we study the thermodynamics of the fundamental matter sector which is dual to the thermal contributions of the D7-branes. According the AdS/CFT dictionary the partition function $Z$ of the boundary field theory is given in terms of the Euclidean on-shell supergravity action $I_{\text {on-shell, }}$

$$
Z=\mathrm{e}^{-I_{\text {on-shell }}}
$$

Thus the thermodynamical potential, i. e. in the grand canonical ensemble the grand potential $\Omega$, is proportional to the Euclidean on-shell supergravity action,

$$
\Omega=-T \ln Z=T I_{\text {on-shell }}
$$


To calculate the thermal contributions of the D7-branes $\Omega_{7}$, we have to determine the Euclidean version of the DBI action (3.12) on-shell.

\subsection{Adapted symmetrized trace prescription}

Now we calculate the Euclidean on-shell action using the adapted symmetrized trace prescription defined in section 3.2. First we perform a Wick rotation in the time direction. Next we renormalize the action by adding appropriate counterterms $I_{\text {ct }}$ (see [49] for a review). Since the action (3.13) has the same structure as the DBI action in $[26,32,50]$ and since in these cases the counterterms do not depend on the finite densities, we can write the counterterms as in $[26,32,50]$,

$$
I_{\mathrm{ct}}=-\frac{\mathcal{N}_{\lambda}}{4}\left[\left(\rho_{\max }^{2}-m^{2}\right)^{2}-4 m c\right],
$$

where $\rho_{\max }$ is the UV-cutoff and

$$
\mathcal{N}_{\lambda}=\frac{T_{D 7} V_{3} \operatorname{vol}\left(S^{3}\right) N_{f} \varrho_{H}^{4}}{4 T}=\frac{\lambda N_{c} M_{f} V_{3} T^{3}}{32},
$$

with Minkowski space volume $V_{3}$. Then the renormalized Euclidean on-shell action $I_{R}$ may simply be written as

$$
\frac{I_{R}}{\mathcal{N}_{\lambda}}=G(m, \tilde{\mu})-\frac{1}{4}\left[\left(\rho_{\min }^{2}-m^{2}\right)^{2}-4 m c\right],
$$

where $\rho_{\text {min }}$ determines the minimal value of the coordinate $\rho$ on the D7-branes, i. e. $\rho_{\min }=1$ for black hole embeddings which we consider exclusively in this paper, and

$$
G(m, \tilde{\mu})=\int_{\rho_{\min }}^{\infty} \mathrm{d} \rho\left(\rho^{3} f \tilde{f}\left(1-\chi^{2}\right) \Upsilon(\rho, \chi, \tilde{A})-\left(\rho^{3}-\rho m\right)\right) .
$$

In figure 4 we plot the dimensionless grand potential $\mathcal{W}_{7}$ defined as

$$
\Omega_{7}=T I_{R}=\frac{\lambda N_{f} N_{c} V_{3} T^{4}}{32} \mathcal{W}_{7} .
$$

We also calculate the specific heat $C_{7}$

$$
C_{7}=-T \frac{\partial^{2} F_{7}}{\partial T^{2}}
$$

where $F_{7}$ is the free energy in the canonical ensemble. ${ }^{2}$ Using (3.26), the expression for the specific heat can be rewritten as

$$
C_{7}=-\frac{\lambda N_{f} N_{c} T^{3}}{32}\left(12 \mathcal{F}_{7}-6 m \frac{\partial \mathcal{F}_{7}}{\partial m}+m^{2} \frac{\partial^{2} \mathcal{F}_{7}}{\partial m^{2}}\right)
$$

with $F_{7}=\left(\lambda N_{f} N_{c} V_{3} T^{4} / 32\right) \mathcal{F}_{7}$. In figure 9 we plot the dimensionless specific heat $\mathcal{C}_{7}$ defined as

$$
C_{7}=\frac{\lambda N_{f} N_{c} T^{3}}{32} \mathcal{C}_{7}
$$

Below we discuss that our results hint towards a boson liquid being present. We also comment on the relation of our results to [51].

\footnotetext{
${ }^{2}$ Note that in [14] we considered the second derivative of the grand potential in the grand canonical ensemble. Although not simply related to the specific heat, that quantity also shows a discontinuity indicating the second order phase transition.
} 


\subsection{Expansion of the DBI action}

The thermodynamical behavior in the case of the expanded DBI action may be determined in the same way as for the adapted symmetrized trace prescription. As mentioned above, the counterterms are needed to regularize the action and do not depend on the gauge fields on the brane. They are needed to regularize the divergence which occurs due to the infinite volume of the AdS space. For vanishing gauge fields on the brane, the action calculated using the adapted symmetrized trace prescription and the action expanded in field strength $F$ coincide. Thus the counterterms discussed above may also be used to regularize the action expanded in $F$. For the renormalized Euclidean action we obtain

$$
\frac{I_{R}}{\mathcal{N}_{\lambda}}=G(m, \tilde{\mu})-\frac{1}{4}\left[\left(\rho_{\min }^{2}-m^{2}\right)^{2}-4 m c\right],
$$

where

$$
G(m, \tilde{\mu})=\int_{\rho_{\min }}^{\infty} \mathrm{d} \rho\left[\sqrt{-G}\left(1+\frac{\mathcal{T}_{2}}{2}-\frac{\mathcal{T}_{4}}{8}\right)-\left(\rho^{3}-\rho m\right)\right],
$$

with $\mathcal{T}_{2}$ and $\mathcal{T}_{4}$ as defined in equations (3.27) and (A.1). In figure 6 we also plot the dimensionless grand potential $\mathcal{W}_{7}$ obtained by the action expanded to fourth order in $F$ and defined as

$$
\Omega_{7}=T I_{R}=\frac{\lambda N_{f} N_{c} V_{3} T^{4}}{32} \mathcal{W}_{7} .
$$

Note that we have not computed the Legendre-transformed action for the DBI action expanded to fourth order due to technical complications discussed in section 3.2.2. Thus we do not have direct access to the quantities in the canonical ensemble, such as the free energy needed to compute the specific heat. A calculation exploiting thermodynamical relations between the ensembles is postponed to future work.

\subsection{Thermodynamical consistency}

In the following we confirm that both definitions of the grand potential, (5.7) and (5.13), give consistent thermodynamics. For this purpose, we have to vary the grand potential with respect to the thermodynamical variables. In the gravity setup this variation is induced by a variation of the gravity fields. Here we only consider a variation of the gauge field $A_{\mu}^{a}$, since the variation of the embedding function $\chi$ is the same as in [26]. Using the equations of motion, the variation of the dimensionless grand potential with respect to the gauge field $A_{\mu}^{a}$ reduces to a boundary term,

$$
\begin{aligned}
\delta \mathcal{W}_{7} & =\left[\frac{\partial \mathcal{L}}{\partial\left(\partial_{\rho} \tilde{A}_{0}^{3}\right)} \delta \tilde{A}_{0}^{3}+\frac{\partial \mathcal{L}}{\partial\left(\partial_{\rho} \tilde{A}_{3}^{1}\right)} \delta \tilde{A}_{3}^{1}\right]_{\rho_{\min }}^{\infty} \\
& =\tilde{p}_{0}^{3}\left(\rho_{\min }\right) \delta \tilde{A}_{0}^{3}\left(\rho_{\min }\right)+\tilde{p}_{3}^{1}\left(\rho_{\min }\right) \delta \tilde{A}_{3}^{1}\left(\rho_{\min }\right)-\tilde{p}_{0}^{3}(\infty) \delta \tilde{A}_{0}^{3}(\infty)-\tilde{p}_{3}^{1}(\infty) \delta \tilde{A}_{3}^{1}(\infty) .
\end{aligned}
$$

Here $\mathcal{L}$ is given by the integrand of the grand potential. Using the asymptotic behavior of the gauge fields $(3.23),(3.24),(3.29),(3.30)$, the contribution of the fields $\tilde{A}_{3}^{1}$ and $\tilde{p}_{3}^{1}$ vanishes and the result coincides with [26],

$$
\delta \mathcal{W}_{7}=-\tilde{p}_{0}^{3}(\infty) \delta \tilde{A}_{0}^{3}(\infty)=-\tilde{d}_{0}^{3} \delta \tilde{\mu} .
$$




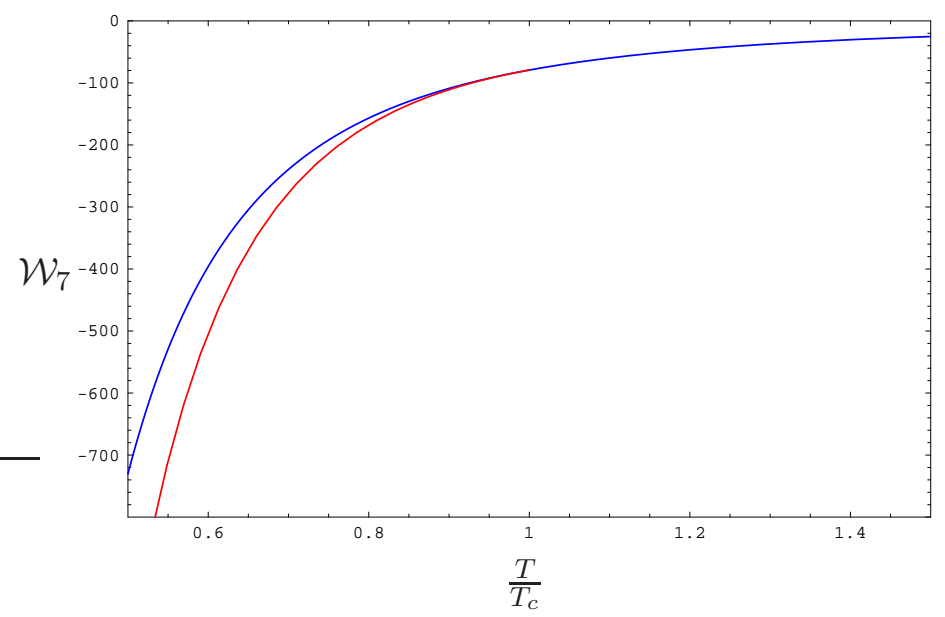

Figure 4. The dimensionless grand canonical potential $\mathcal{W}_{7}$ calculated using the adapted symmetrized trace prescription versus temperature at zero quark mass $M_{q}=0$ : Below $T=T_{c}$ the superconducting phase (red line) is thermodynamically preferred over the normal phase (blue line).

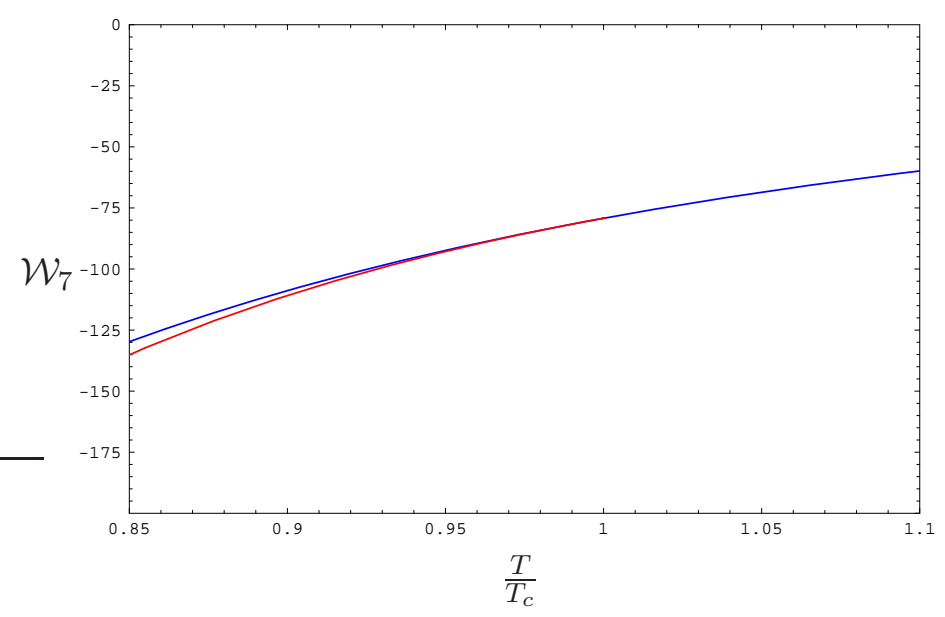

Figure 5. Same as figure 4 close to the phase transition.

Therefore we confirm that $\Omega_{7}$ is the thermodynamical potential in the grand canonical ensemble, namely the grand potential. We also see that the density $\tilde{d}_{3}^{1}$ is not a thermodynamical variable.

\subsection{Results \& comparison of the two prescriptions}

We now compare the results obtained by evaluating the non-Abelian DBI action using the adapted symmetrized trace prescription or the expansion to fourth order.

Figure 4 shows that for non-zero gauge field $A_{3}^{1}$, a phase transition occurs. The presence of this phase transition does not depend on the prescription used, as seen from the comparison of the potential derived using the adapted symmetrized trace prescription (see figure 5) with the results derived from the expanded DBI action (see figure 6). The transition is second order since there is a discontinuous step in the specific heat $\mathcal{C}_{7}$, see figure 9. 


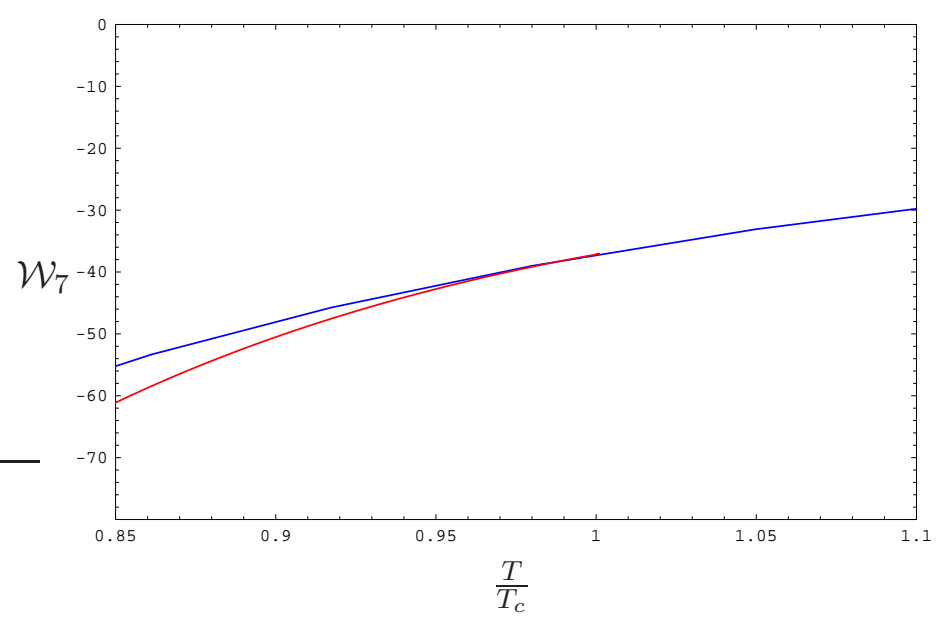

Figure 6. Grand canonical potential computed from the expanded DBI action at vanishing quark mass $M_{q}=0$. The qualitative behavior is in agreement with the results from the adapted symmetrized trace prescription (see figure 5).

The temperature scale in the massless case is defined by

$$
\frac{T}{T_{c}}=\frac{\tilde{\mu}_{c}}{\tilde{\mu}}=\left(\frac{\left(\tilde{d}_{0}^{3}\right)_{c}}{\tilde{d}_{0}^{3}}\right)^{\frac{1}{3}},
$$

where $\tilde{\mu}_{c} \approx 2.85$ and $\left(\tilde{d}_{0}^{3}\right)_{c} \approx 20.7$ as obtained from the adapted symmetrized trace prescription (see section 3.2.1). For the expanded DBI action we equivalently obtain $\tilde{\mu}_{c} \approx 2.48$ and $\left(\tilde{d}_{0}^{3}\right)_{c} \approx 9.03$. In the massive case we fix a quark mass $M_{q}$, then a chemical potential $\mu$ measured in units of $M_{q}$. Thus the temperature scale is defined as

$$
\frac{T}{T_{c}}=\frac{m_{c}}{m}
$$

where $m_{c}$ is the value of the mass parameter at which the phase transition occurs.

From the behavior of the order parameter $\tilde{d}_{3}^{1}$, see in figure 7 , near the critical temperature, we obtain the critical exponent of the transition to be $1 / 2$, which coincides with the result obtained from Landau theory. It is also determined numerically from a fit within 10 percent accuracy, see blue dashed curve in figure 7 . This exponent is independent from the quark mass as seen from the black curve at $\mu / M_{q}=3$ shown in figure 7 . We have also confirmed numerically that the critical exponent of the order parameter $\tilde{d}_{3}^{1}$ does not depend on the prescription (adapted symmetrized trace or expanded DBI). Since the order parameter $\tilde{d}_{3}^{1}$ and the density $\tilde{d}_{0}^{3}$ increase rapidly as $T \rightarrow 0$, we expect that the probe approximation breaks down near $T=0$.

We define the density of superconducting charges $\tilde{d}_{s}$ in the following way

$$
\tilde{d}_{s}=\frac{\tilde{p}_{0}^{3}\left(\rho_{B}\right)-\tilde{p}_{0}^{3}\left(\rho_{H}\right)}{\tilde{p}_{0}^{3}\left(\rho_{B}\right)}=\frac{\tilde{d}_{0}^{3}-c_{0}}{\tilde{d}_{0}^{3}} .
$$

This identification was suggested in [7]. Beyond the arguments given there, we have the advantage of exactly knowing the dual field theory interpretation and having a string 


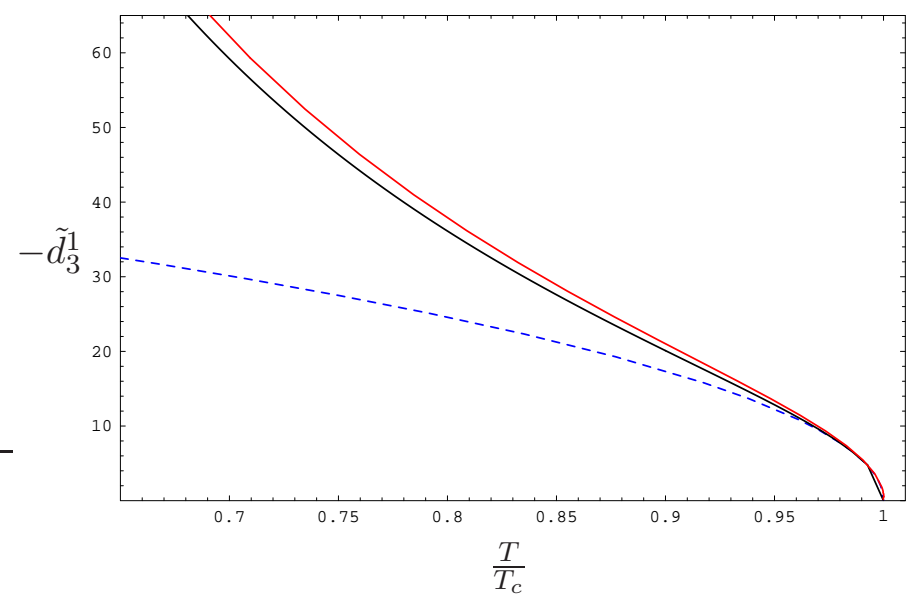

Figure 7. The order parameter $\tilde{d}_{3}^{1}$ defined in (3.24) and obtained from the adapted symmetrized trace prescription versus temperature $T$ : The case of vanishing quark mass (red curve) shows the same behavior near $T_{c}$ as that at finite mass where $\mu / M_{q}=3$ is fixed. In both cases the order parameter vanishes with a critical exponent of $1 / 2$. This is visualized by the fit $55\left(1-T / T_{c}\right)^{1 / 2}$ (dashed blue curve).

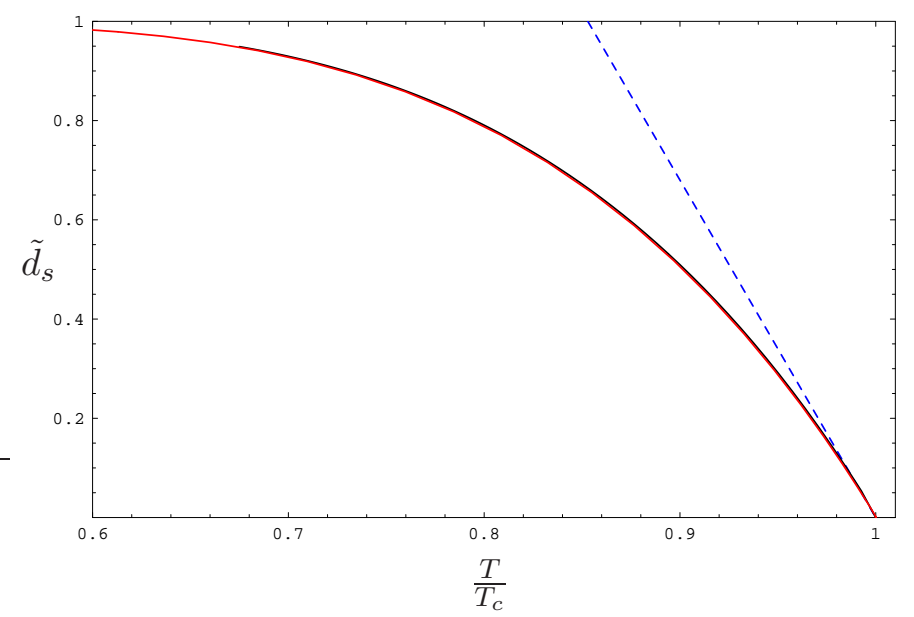

Figure 8. Superconducting density $\tilde{d}_{s}=\left(\tilde{d}_{0}^{3}-c_{0}\right) / \tilde{d}_{0}^{3}$ versus temperature $T$ : In both, the massless (red curve) and the massive case at $\mu / M_{q}=3$ (blue curve), the superconducting density $\tilde{d}_{s}$ vanishes linearly at the critical temperature. This is visualized by the fit $6.8\left(1-T / T_{c}\right)$ (dashed blue curve).

picture, see section 4. The numerator of equation (5.18) counts the isospin charges present in the bulk excluding those localized at the black hole horizon $p_{0}^{3}\left(\rho_{H}\right)=c_{0}$. Following the ideas described in section 4 , these charges may be identified with the D7-D7 strings in the bulk, which are dual to the Cooper pairs. As expected from Ginzburg-Landau theory, this superconducting density $\tilde{d}_{s}$ vanishes linearly at $T_{c}$ in massive and massless cases regardless of the prescription chosen, see figure 8 .

In figure 9 the dimensionless specific heat computed from the adapted symmetrized trace prescription for massless quarks is shown versus the temperature. It clearly shows a discontinuity at the phase transition, stressing that the observed transition is second 


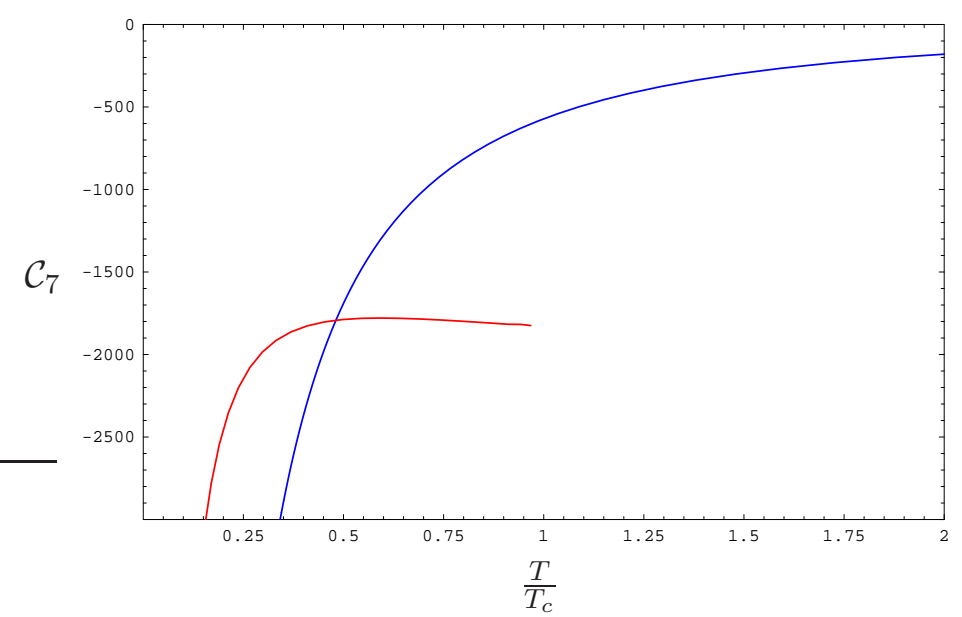

Figure 9. The flavor brane contribution to the specific heat as computed from the adapted symmetrized trace prescription in the massless case. The blue line corresponds to the normal phase with $A_{3}^{1}=0$, while the red one corresponds to the superconducting phase with $A_{3}^{1} \neq 0$. Note that the total specific heat is always positive although the flavor brane contribution is negative. The divergences near $T=0$ in both phases can be attributed to the missing backreaction in our setup. We read of from the numerical result that near the critical temperature, the dimensionless specific heat is constant in the superconducting phase. This implies that the dimensionful specific heat is proportional to $T^{3}$. This temperature dependence is characteristic for Bose liquids.

order. Note that the dimensionful specific heat is given by $C_{7} \propto T^{3} \mathcal{C}_{7}$, where $\mathcal{C}_{7}$ is the dimensionless specific heat shown in figure 9 .

The blue curve in figure 9 corresponds to the normal phase while the red one shows the behavior of the superconducting phase. A general feature of superfluids, including charged superfluids as in the present case, is the behavior of the specific heat near $T=0$. For Bose liquids the heat capacity has cubic temperature dependence. In Fermi liquids, a linear temperature dependence dominates near $T=0$. In our numerical results (figure 9) we see a dramatic change of specific heat in the normal phase (blue curve) in sharp contrast to the constant behavior, corresponding to $C_{7} \propto T^{3}$, of the superconducting phase (red curve) close to the critical temperature. The divergent behavior close to $T=0$ is due to the probe approximation which we use. Although our numerics do not allow to extend the calculation up to $T=0$, it is tempting to extrapolate the observed behavior towards $T=0$ and to conclude that our setup in the superconducting phase is a Bose liquid.

Recently a new type of quantum liquid has been discussed in [51], where the authors consider a brane setup at zero mass in the limit of infinite baryon density, i. e. at zero temperature. In that case the heat capacity was found to be proportional to $T^{6}$, neither typical for a Fermi nor for a Bose liquid.

In the following we argue that our result found above suggests to consider the possibility of a phase transition also in the baryonic setup [51]. The baryonic setup of [51] may be related to our normal phase by a symmetry between pure baryonic and pure isospin configurations found in [32]. Thus the specific heat in the baryonic setup and in our normal-conducting phase coincide. Since in our isospin case there is a phase transition 


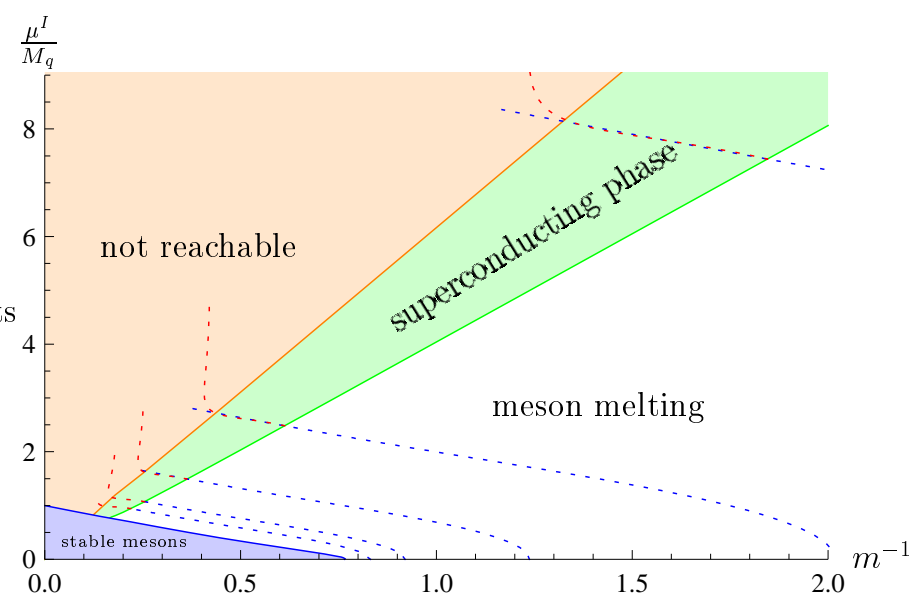

Figure 10. Phase diagram for fundamental matter with mass $m=2 M_{q} /(\sqrt{\lambda} T)$ obtained with the adapted symmetrized trace prescription: The blue, white and green regions are the same as in figure 1, but with the unstable normal phase replaced with the superconducting phase. The dotted curves correspond to lines at finite mass, i. e. constant $\chi_{0}$. These curves are parametrized by the density $\tilde{d}_{0}^{3}$. Along the blue curves the field $A_{3}^{1}$ is zero while along the red ones the field $A_{3}^{1}$ is non-zero. The endpoints of the red curves determine the second order phase transition to the superconducting phase. The dotted, red curves diverge inside the superconducting phase since the backreaction of the condensate on the background is not considered. This divergence determines the boundary of the orange region which is not reachable without backreaction.

to the superconducting phase which shows the usual Bose liquid behavior, we suggest the possibility of a similar phase transition in the baryonic setup of [51]. The possible new phase may lead to the usual behavior of a Bose or Fermi liquid.

Summarizing our thermodynamical results in a phase diagram, we obtain figure 10 . The choice of the calculational method - adapted symmetrized trace or expansion of the DBI to fourth order - does not change the qualitative structure of the phase diagram. The blue phase indicates the known region of stable mesons surviving the deconfinement transition. It is separated from the white meson melting region by the meson melting transition (blue line), see [25-27, 29, 32, 50, 52, 53]. Above a critical isospin density marked by the green line, a flavor-superconducting phase forms. At even higher isospin density, our approach, which does not include the back-reaction of the D7-brane, gives diverging order parameters, signalling the breakdown of this approach. This particular region is indicated by orange color. Note that this behavior is well-known to occur in systems without backreaction. In our case, the gauge fields on the D7-brane grow arbitrarily large close to zero temperature. This behavior will be cured by including the back-reaction, i.e. the contribution of the gauge fields to the total energy-momentum tensor. For the Abelian Higgs model, it is shown in [13] that similar divergences are removed when these terms are included. 


\section{$6 \quad$ Fluctuations}

The full gauge field $\hat{A}$ on the branes consists of the field $A$ and fluctuations $a$,

$$
\hat{A}=A_{0}^{3} \tau^{3} \mathrm{~d} t+A_{3}^{1} \tau^{1} \mathrm{~d} x_{3}+a_{\mu}^{a} \tau^{a} \mathrm{~d} x^{\mu},
$$

where $\tau^{a}$ are the $\mathrm{SU}(2)$ generators. The linearized equations of motion for the fluctuations $a$ are obtained by expanding the DBI action in $a$ to second order. We will analyze the fluctuations $a_{2}^{3}$ and $X=a_{2}^{1}+\mathrm{i} a_{2}^{2}, Y=a_{2}^{1}-\mathrm{i} a_{2}^{2}$.

Including these fluctuations, the DBI action reads

$$
S=-T_{7} \int \mathrm{d}^{8} \xi \operatorname{Str} \sqrt{\operatorname{det}\left[G+\left(2 \pi \alpha^{\prime}\right) \hat{F}\right]},
$$

with the non-Abelian field strength tensor

$$
\hat{F}_{\mu \nu}^{a}=F_{\mu \nu}^{a}+\check{F}_{\mu \nu}^{a},
$$

where the background is collected in

$$
F_{\mu \nu}^{a}=2 \partial_{[\mu} A_{\nu]}^{a}+\frac{\gamma}{\sqrt{\lambda}} f^{a b c} A_{\mu}^{b} A_{\nu}^{c},
$$

and all terms containing fluctuations in the gauge field are summed in

$$
\check{F}_{\mu \nu}^{a}=2 \partial_{[\mu} a_{\nu]}^{a}+\frac{\gamma}{\sqrt{\lambda}} f^{a b c} a_{\mu}^{b} a_{\nu}^{c}+\frac{\gamma}{\sqrt{\lambda}} f^{a b c}\left(A_{\mu}^{b} a_{\nu}^{c}+a_{\mu}^{b} A_{\nu}^{c}\right) .
$$

Index anti-symmetrization is always defined with a factor of two in the following way $\partial_{[\mu} A_{\nu]}=\left(\partial_{\mu} A_{\nu}-\partial_{\nu} A_{\mu}\right) / 2$.

\subsection{Adapted symmetrized trace prescription}

In this section we use the adapted symmetrized trace prescription to determine the fluctuations about the background we discussed in section 3.2.1. To obtain the linearized equations of motion for the fluctuations $a$, we expand the action (6.2) to second order in fluctuations,

$$
\begin{aligned}
S^{(2)}=-T_{7} \int \mathrm{d}^{8} \xi \operatorname{Str}[ & \sqrt{-\mathcal{G}}+\frac{\left(2 \pi \alpha^{\prime}\right)}{2} \sqrt{-\mathcal{G}} \mathcal{G}^{\mu \nu} \check{F}_{\nu \mu}-\frac{\left(2 \pi \alpha^{\prime}\right)^{2}}{4} \sqrt{-\mathcal{G}} \mathcal{G}^{\mu \mu^{\prime}} \check{F}_{\mu^{\prime} \nu} \mathcal{G}^{\nu \nu^{\prime}} \check{F}_{\nu^{\prime} \mu} \\
& \left.+\frac{\left(2 \pi \alpha^{\prime}\right)^{2}}{8} \sqrt{-\mathcal{G}}\left(\mathcal{G}^{\mu \nu} \check{F}_{\nu \mu}\right)^{2} g\right]
\end{aligned}
$$

As in the previous paper [33], we collect the metric and gauge field background in the tensor $\mathcal{G}=G+\left(2 \pi \alpha^{\prime}\right) F$. Using the Euler-Lagrange equation, we get the linearized equation of motion for fluctuations $a_{\kappa}^{d}$ in the form

$$
\begin{aligned}
0= & \partial_{\lambda} \operatorname{Str}\left[\sqrt{-\mathcal{G}} \tau^{d}\left\{\mathcal{G}^{[\kappa \lambda]}+\left(2 \pi \alpha^{\prime}\right)\left(\mathcal{G}^{\mu[\kappa} \mathcal{G}^{\lambda] \nu}+\frac{1}{2} \mathcal{G}^{\mu \nu} \mathcal{G}^{[\kappa \lambda]}\right) \check{F}_{\nu \mu}\right\}\right] \\
& -\operatorname{Str}\left[\frac{c}{\sqrt{\lambda}} f^{a b d} \tau^{a} \sqrt{-\mathcal{G}}\left\{\mathcal{G}^{[\kappa \lambda]}(a+A)_{\lambda}^{b}+\left(2 \pi \alpha^{\prime}\right)\left(\mathcal{G}^{\mu[\kappa} \mathcal{G}^{\lambda] \nu}+\frac{1}{2} \mathcal{G}^{\mu \nu} \mathcal{G}^{[\kappa \lambda]}\right) \check{F}_{\nu \mu} A_{\lambda}^{b}\right\}\right] .
\end{aligned}
$$


Note that the linearized version of the fluctuation field strength used in equation (6.7) is given by

$$
\check{F}_{\mu \nu}^{a}=2 \partial_{[\mu} a_{\nu]}^{a}+\frac{\gamma}{\sqrt{\lambda}} f^{a b c}\left(A_{\mu}^{b} a_{\nu}^{c}+a_{\mu}^{b} A_{\nu}^{c}\right)+\mathcal{O}\left(a^{2}\right) .
$$

In our specific case the background tensor in its covariant form is given by

$$
\mathcal{G}_{\mu \nu}=G_{\mu \nu} \tau^{0}+\left(2 \pi \alpha^{\prime}\right)\left(2 \partial_{\varrho} A_{0}^{3} \delta_{4[\mu} \delta_{\nu] 0} \tau^{3}+2 \partial_{\varrho} A_{3}^{1} \delta_{4[\mu} \delta_{\nu] 3} \tau^{1}+2 \frac{\gamma}{\sqrt{\lambda}} A_{0}^{3} A_{3}^{1} \delta_{0[\mu} \delta_{\nu] 3} \tau^{2}\right) .
$$

Inversion yields the contravariant form needed to compute the explicit equations of motion. The inverse of $\mathcal{G}$ is defined as $\mathcal{G}^{\mu \nu} \mathcal{G}_{\nu \mu^{\prime}}=\delta_{\mu^{\prime}}^{\mu} \tau^{0}{ }^{3}$. The non-zero components of $\mathcal{G}^{\mu \nu}$ may be found in the appendix B.1.

Fluctuations in $\boldsymbol{a}_{2}^{3}$ : for the fluctuation $a_{2}^{3}$ with zero spatial momentum, we obtain the equation of motion

$$
\left(a_{2}^{3}\right)^{\prime \prime}+\frac{\partial_{\rho} H}{H}\left(a_{2}^{3}\right)^{\prime}-\left[\frac{4 \varrho_{H}^{4}}{R^{4}}\left(\frac{\mathcal{G}^{33}}{\mathcal{G}^{44}}\left(\mathfrak{m}_{3}^{1}\right)^{2}+\frac{\mathcal{G}^{00}}{\mathcal{G}^{44}} \mathfrak{w}^{2}\right)-16 \frac{\partial_{\rho}\left(\frac{H}{\rho^{4} f^{2}} \tilde{A}_{0}^{3}\left(\partial_{\rho} \tilde{A}_{0}^{3}\right)\left(\mathfrak{m}_{3}^{1}\right)^{2}\right)}{H\left(1-\frac{2 c^{2}}{\pi^{2} \rho^{4} f^{2}}\left(\tilde{A}_{3}^{1} \tilde{A}_{0}^{3}\right)^{2}\right)}\right] a_{2}^{3}=0,
$$

with

$$
\mathfrak{m}_{3}^{1}=\frac{\gamma}{2 \sqrt{2} \pi} \tilde{A}_{3}^{1}, \quad H=\sqrt{\mathcal{G}} G^{22} \mathcal{G}^{44} .
$$

Fluctuations in $X=\boldsymbol{a}_{2}^{1}+\mathbf{i} \boldsymbol{a}_{2}^{2}, \boldsymbol{Y}=\boldsymbol{a}_{2}^{1}-\mathbf{i} \boldsymbol{a}_{2}^{2}$ : for the fluctuations $X$ and $Y$ with zero spatial momentum, we obtain the coupled equations of motion

$$
\begin{aligned}
0= & X^{\prime \prime}+\frac{\partial_{\rho} H}{H} X^{\prime}-\frac{4 \varrho_{H}^{4}}{R^{4}}\left[\frac{\mathcal{G}^{00}}{\mathcal{G}^{44}}\left(\mathfrak{w}-\mathfrak{m}_{0}^{3}\right)^{2}+\frac{\mathcal{G}^{\{03\}}}{\mathcal{G}^{44}} \mathfrak{m}_{3}^{1} \mathfrak{w}\right] X+\frac{4 \varrho_{H}^{4}}{R^{4}}\left[\frac{\mathcal{G}^{\{03\}}}{\mathcal{G}^{44}} \mathfrak{m}_{3}^{1} \mathfrak{m}_{0}^{3}\right. \\
& \left.+\frac{R^{2}}{4 \varrho_{H}^{2}} \frac{\partial_{\rho}\left[\sqrt{-\mathcal{G}} G^{22} \mathcal{G}^{\{34\}} \mathfrak{m}_{3}^{1}\right]}{H}-\frac{\mathcal{G}^{33}}{2 \mathcal{G}^{44}}\left(\mathfrak{m}_{3}^{1}\right)^{2}\right](X-Y)+\frac{4 \varrho_{H}^{2}}{R^{2}} \frac{\mathcal{G}^{\{04\}}}{\mathcal{G}^{44}} \mathfrak{w} Y^{\prime} \\
& +\frac{2 \varrho_{H}^{2}}{R^{2}} \frac{\partial_{\rho}\left[\sqrt{-\mathcal{G}} G^{22} \mathcal{G}^{\{04\}}\left(\mathfrak{w}+\mathfrak{m}_{0}^{3}\right)\right]}{H} Y, \\
0 & Y^{\prime \prime}+\frac{\partial_{\rho} H}{H} Y^{\prime}+\frac{4 \varrho_{H}^{4}}{R^{4}}\left[-\frac{\mathcal{G}^{00}}{\mathcal{G}^{44}}\left(\mathfrak{w}+\mathfrak{m}_{0}^{3}\right)^{2}+\frac{\mathcal{G}^{\{03\}}}{\mathcal{G}^{44}} \mathfrak{m}_{3}^{1} \mathfrak{w}\right] Y-\frac{4 \varrho_{H}^{4}}{R^{4}}\left[\frac{\mathcal{G}^{\{03\}}}{\mathcal{G}^{44}} \mathfrak{m}_{3}^{1} \mathfrak{m}_{0}^{3}\right. \\
& \left.+\frac{R^{2}}{4 \varrho_{H}^{2}} \frac{\partial_{\rho}\left[\sqrt{-\mathcal{G}} G^{22} \mathcal{G}^{\{34\}} \mathfrak{m}_{3}^{1}\right]}{H}-\frac{\mathcal{G}^{33}}{2 \mathcal{G}^{44}}\left(\mathfrak{m}_{3}^{1}\right)^{2}\right](X-Y)-\frac{4 \varrho_{H}^{2}}{R^{2}} \frac{\mathcal{G}^{\{04\}}}{\mathcal{G}^{44}} \mathfrak{w} X^{\prime} \\
& -\frac{2 \varrho_{H}^{2}}{R^{2}} \frac{\partial_{\rho}\left[\sqrt{-\mathcal{G}} G^{22} \mathcal{G}^{\{04\}}\left(\mathfrak{w}-\mathfrak{m}_{0}^{3}\right)\right]}{H} X,
\end{aligned}
$$

where the component of the inverse background tensor may be found in the appendix B.1, index symmetrization is defined $\mathcal{G}^{\{i j\}}=\left(\mathcal{G}^{i j}+\mathcal{G}^{j i}\right) / 2$ and

$$
\mathfrak{m}_{0}^{3}=\frac{\gamma}{2 \sqrt{2} \pi} \tilde{A}_{0}^{3} .
$$

\footnotetext{
${ }^{3}$ We calculate the inverse of $\mathcal{G}$ by ignoring the commutation relation of the $\tau$ 's because of symmetrized trace. It is important that $\tau^{a} \tau^{b}$ must not be simplified to $\epsilon^{a b c} \tau^{c}$ since symmetrisation is not the same.
} 


\subsection{Expansion of the DBI action}

In this section we determine the equation of motion for the fluctuation $a_{2}^{3}$ in the background determined by the DBI action expanded to fourth order in $F$ (see section 3.2.2). To obtain the quadratic action in the field $a_{2}^{3}$, we first have to expand the DBI action (6.2) to fourth order in the full gauge field strength $\hat{F}$, and expand the result to second order in $a_{2}^{3}$. Due to the symmetries of our setup, the equation of motion for the fluctuation $a_{2}^{3}$ at zero spatial momentum decouples from the other equations of motion, such that we can write down an effective Lagrangian for the fluctuation $a_{3}^{2}$. This effective Lagrangian is given in the appendix B.2. The equation of motion for $a_{2}^{3}$ with zero spatial momentum determined by the Euler-Lagrange equation is given by

$$
\begin{aligned}
0=\left(a_{2}^{3}\right)^{\prime \prime}+\frac{\partial_{\rho} \mathcal{H}}{\mathcal{H}}\left(a_{2}^{3}\right)^{\prime}-\frac{\varrho_{H}^{4}}{R^{4}}\left[4\left(\frac{\mathcal{H}^{00}}{\mathcal{H}^{44}} \mathfrak{w}^{2}+\frac{\mathcal{H}^{33}}{\mathcal{H}^{44}}\left(\mathfrak{m}_{3}^{1}\right)^{2}\right)\right. \\
\left.+\frac{8}{3} \frac{\partial_{\rho}\left[\sqrt{-G} G^{00} G^{22} G^{33} G^{44} \tilde{A}_{0}^{3}\left(\partial_{\rho} \tilde{A}_{0}^{3}\right)\left(\mathfrak{m}_{3}^{1}\right)^{2}\right]}{\mathcal{H}}\right] a_{2}^{3},
\end{aligned}
$$

where

$$
\mathcal{H}=\sqrt{-G} G^{22} \mathcal{H}^{44} .
$$

We introduce the factors $\mathcal{H}^{i j}$ which may be found in the appendix B.2 to emphasize the similarity to the equation of motion obtained by the adapted symmetrized trace prescription (6.10).

\subsection{Result \& comparison of the two prescriptions}

\subsubsection{Conductivity}

We calculate the frequency-dependent conductivity $\sigma(\omega)$ using the Kubo formula,

$$
\sigma(\omega)=\frac{\mathrm{i}}{\omega} G^{R}(\omega, q=0),
$$

where $G^{R}$ is the retarded Green function of the current $J_{2}^{3}$ dual to the fluctuation $a_{2}^{3}$, which we calculate using the method obtained in [54]. The current $J_{2}^{3}$ is the analog to the electric current since it is charged under the $\mathrm{U}(1)_{3}$ symmetry. In real space it is transverse to the condensate. Since this fluctuation is the only one which transforms as a vector under the $\mathrm{SO}(2)$ rotational symmetry, it decouples from the other fluctuations of the system.

The real part of the frequency-dependent conductivity $\operatorname{Re} \sigma(\omega)$ is presented in figure $11,12,13,15$. It shows the appearance and growth of a gap as we increase the condensate $\tilde{d}_{3}^{1}$. The conductivity gap originates in a pseudo gap already present right above $T_{c}$, as can be seen for example from the red curve in figure 11. By pseudo gap we mean a well-defined gap in the conductivity at low frequency in which the conductivity is not identically zero [8]. Both calculational prescriptions - the adapted symmetrized trace prescription and the expansion of the DBI action to fourth order in the field strength - yield qualitatively very similar results as can be seen by comparing figures 13 and 15 . As a further distinct effect when using the adapted symmetrized trace prescription, figure 11, 12, 13 show prominent peaks which we interpret as mesonic excitations below. 


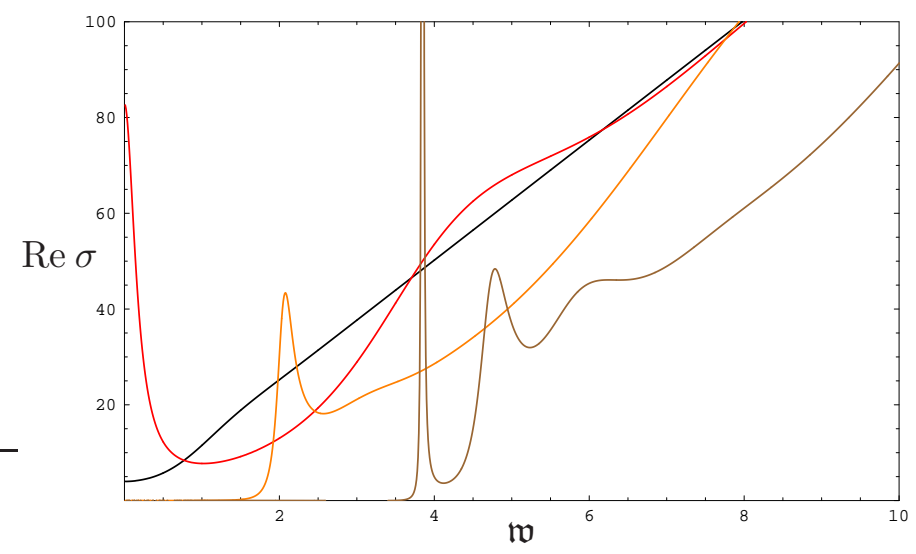

Figure 11. Real part of conductivity, $\operatorname{Re} \sigma$, in units of $N_{f} N_{c} T /(16 \pi)$ versus the dimensionless frequency $\mathfrak{w}=\omega /(2 \pi T)$ for massless quarks computed from the adapted symmetrized trace prescription. Distinct curves correspond to $T / T_{c}=\infty$ (black), 1 (red), 0.5 (orange) and 0.28 (brown). By decreasing the temperature below the critical one, a gap where the conductivity is approximately zero appears which is a characteristic feature of a superconductor. In addition prominent peaks arise.

Increasing the quark mass $M_{q}$ from zero to a finite value, these meson peaks become sharper, i.e. more quasiparticle-like. This is reminiscent of results for condensed matter systems where prominent quasiparticle peaks appear (e.g. [55]). In figure 12 at fixed $\mu / M_{q}=3$, the sharp resonances are also present inside the gap. In contrast to the adapted symmetrized trace prescription, from the expanded DBI action we obtain less prominent peaks as seen from figure 15. In the conductivity obtained from the DBI action expanded to fourth order, the peaks do not appear until we approach small temperatures. We expect that the terms higher order in the field strength dominate the generation of the peaks and therefore the generation of the meson mass. If the higher order terms discussed here are included, we presume that the quasinormal modes which generate these peaks move closer to the real axis.

Using the Kramers-Kronig relation, which connects the real and imaginary part of the complex conductivity, we find a delta peak at $\omega=0$ in the real part of the conductivity, $\operatorname{Re} \sigma(\omega) \sim \pi n_{s} \delta(\omega)$. The corresponding $n_{s} / \omega$-behavior in the imaginary part is visualized in figure 14. As expected from Ginzburg-Landau theory, our numerics show that the superconducting density $n_{s}$ vanishes linearly at the critical temperature, $n_{s} \propto\left(1-T / T_{c}\right)$ for $T \approx T_{c}$. This field theory definition of the superconducting density $n_{s}$ yields a quantity with the same linear scaling near $T_{c}$ as found in our bulk definition (5.18) of the superconducting density $\tilde{d}_{s}$. This confirms that these two quantities may be identified. Our numerics indeed indicate that $\tilde{d}_{s}$ and $n_{s}$ are proportional to each other.

Note that for translation invariant systems at finite density, there is a delta peak in the real part of the conductivity even in a normal conducting phase since the charge carriers cannot lose their momentum. This peak is called Drude peak. In our system, however, the charge carriers can dissipate their momentum although our system is translation invariant $[56,57]$. The adjoint degrees of freedom can transfer momentum at order $N_{c}^{2}$ while 


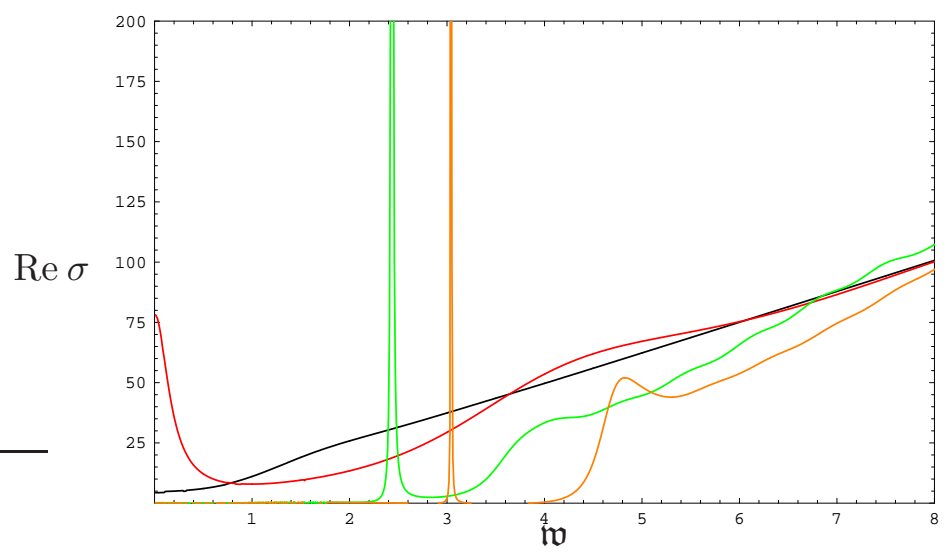

Figure 12. Real part of conductivity, Re $\sigma$, in units of $N_{f} N_{c} T /(16 \pi)$ versus the dimensionless frequency $\mathfrak{w}=\omega /(2 \pi T)$ for massive quarks at fixed $\mu / M_{q}=3$ computed from the adapted symmetrized trace prescription. Distinct curves correspond to $T / T_{c}=10$ (black), 1(red), 0.6 (green) and 0.5 (orange). As in figure 11 a gap appears. Moreover, we observe a prominent peak inside the gap where the conductivity is approximately zero in the green and orange curves.

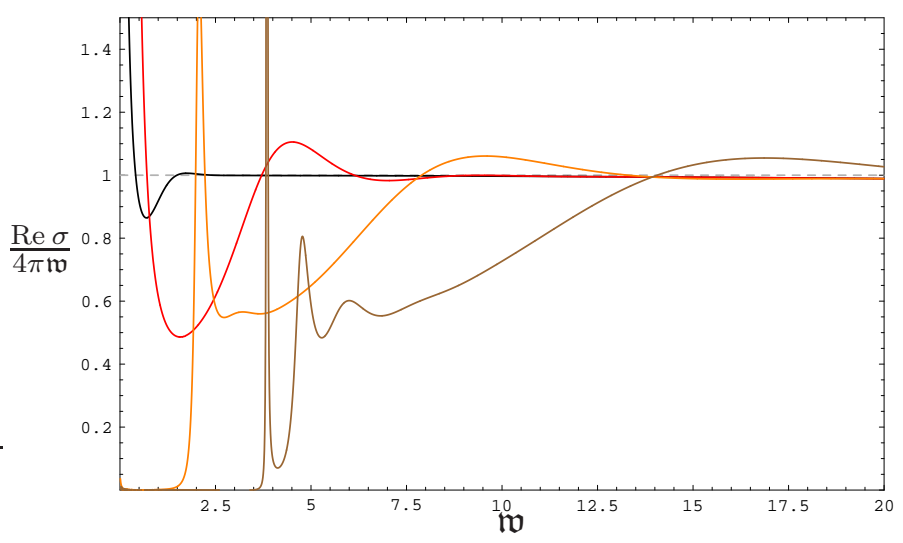

Figure 13. Scaled real part of conductivity $\operatorname{Re} \sigma /(4 \pi \mathfrak{w})$ in units of $N_{f} N_{c} T /(16 \pi)$ versus the dimensionless frequency $\mathfrak{w}=\omega /(2 \pi T)$ for massive quarks at fixed $\mu / M_{q}=3$ computed from the adapted symmetrized trace prescription. Distinct curves correspond to $T / T_{c}=10$ (black), 1(red), 0.5 (orange) and 0.28 (brown). This figure has been scaled to asymptote to a constant in order to show similarity to the lower dimensional cases computed from $A d S_{4}$ and to show common asymptotics.

the fundamental degrees of freedom only at order $N_{c}$. The adjoint degrees of freedom effectively act as a heat sink into which the flavor fields can dissipate their momentum. Thus we do not observe a Drude peak in our system.

\subsubsection{Spectral functions}

In order to identify the prominent peaks in the conductivity in terms of field theory quantities as meson resonances, we examine the spectral functions $\mathcal{R}=-2 \operatorname{Im} G^{R}$ which are related to these conductivities by (6.17). Due to this relation the prominent peaks in the conductivity correspond to the prominent peaks in the spectral function, see figure 16. As known from $[33,58]$ the resonances appearing in the spectral functions of vector fields in 


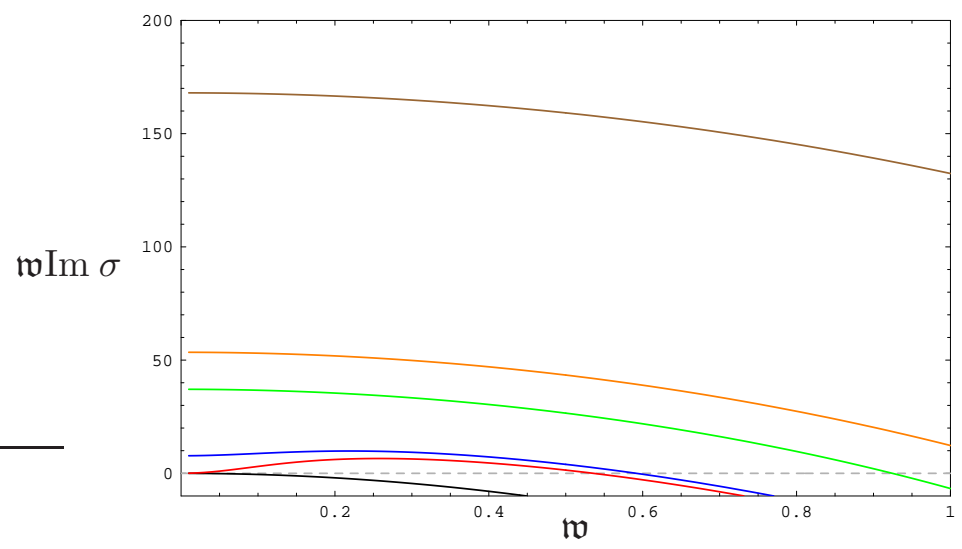

Figure 14. Scaled imaginary part of conductivity $\mathfrak{w} \operatorname{Im} \sigma$ in units of $N_{f} N_{c} T /(16 \pi)$ versus the dimensionless frequency $\mathfrak{w}=\omega /(2 \pi T)$ for massless quarks computed from the adapted symmetrized trace prescription. Distinct curves correspond to $T / T_{c}=\infty$ (black), 1 (red), 0.9 (blue), 0.6 (green), 0.5 (orange) and 0.28 (brown). This figure has been scaled to asymptote to a constant at $\mathfrak{w}=0$. This constant determines the superconducting density $n_{s}$.

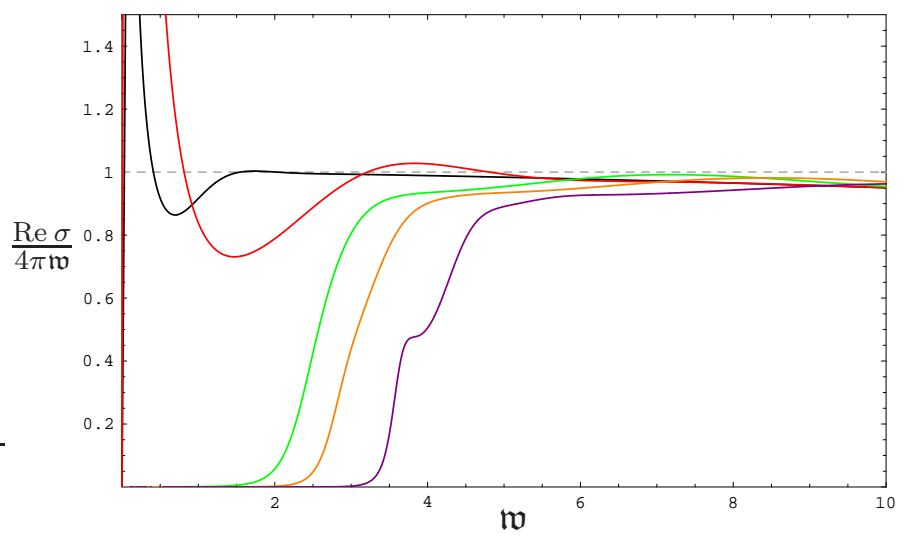

Figure 15. Scaled real part of conductivity $\operatorname{Re} \sigma /(4 \pi \mathfrak{w})$ in units of $N_{f} N_{c} T /(16 \pi)$ versus the dimensionless frequency $\mathfrak{w}=\omega /(2 \pi T)$ for massless quarks computed from the expanded DBI action. Distinct curves correspond to $T / T_{c}=\infty$ (black), 1 (red), 0.6 (green), 0.5 (orange) and 0.39 (purple). This figure has been scaled to asymptote to a constant in order to show similarity to the lower dimensional cases computed from $A d S_{4}$ and to show common asymptotics.

the bulk correspond to vector meson excitations in the dual field theory. Moving to higher quark mass parameter $m=2.842$ and $\tilde{\mu}=3.483$ near the meson melting transition, we compare the resulting spectrum, see figure 17 to the supersymmetric mass formula obtained in [24]. The prominent peaks clearly approach the supersymmetric line spectrum from above. The same behavior was found in figure 7 of [33], where vector mesons were considered also close to the meson melting transition. Note that the accuracy of our numerics becomes insufficient at larger frequencies around $\mathfrak{w} \approx 15 .{ }^{4}$

\footnotetext{
${ }^{4}$ The frequency range of validity of our fluctuation numerics increases with quark mass. However, the numerics for the background become worse at large quark masses.
} 


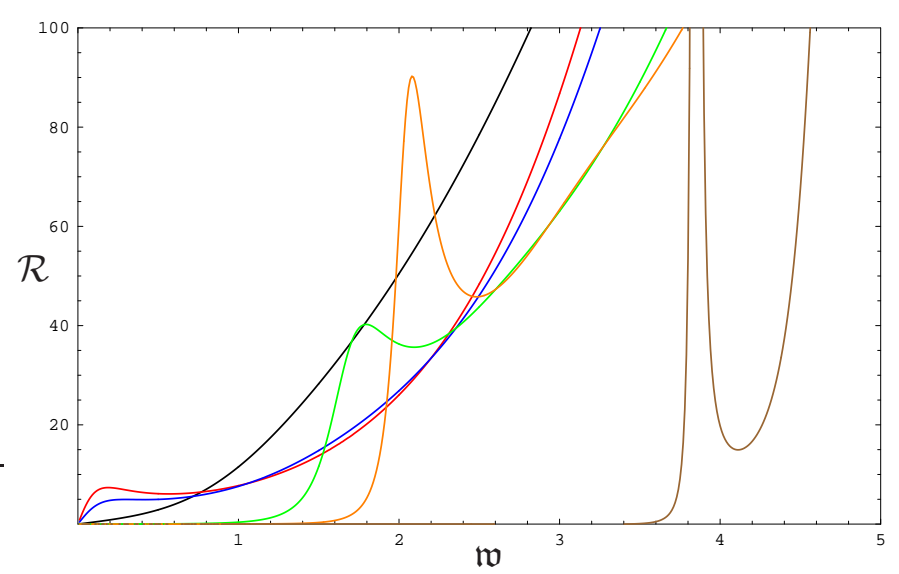

Figure 16. The spectral function $\mathcal{R}$ in units of $N_{f} N_{c} T^{2} / 8$ versus the dimensionless frequency $\mathfrak{w}=\omega /(2 \pi T)$ for massless quarks $M_{q}=0$. The distinct curves corresponds to different temperature (for color coding see figure 14). When decreasing the temperature, peaks which correspond to mesonic quasiparticles emerge.

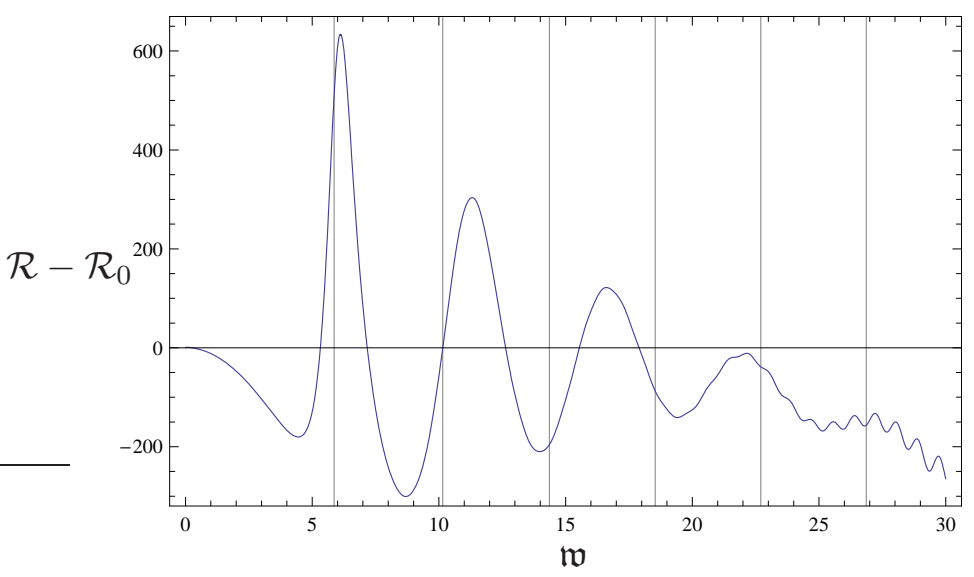

Figure 17. Finite temperature part of the spectral function $\mathcal{R}-\mathcal{R}_{0}$ with $\mathcal{R}_{0}=4 \pi \mathfrak{w}^{2}$ in units of $N_{f} N_{c} T^{2} / 8$ versus the dimensionless frequency $\mathfrak{w}=\omega /(2 \pi T)$ at finite mass $m=2.842$ and chemical potential $\tilde{\mu}=3.483$. The grey lines correspond to the supersymmetric mass spectrum calculated in [24].

\subsubsection{Comments on stability}

As shown in figure 18, our setup is stable with respect to the fluctuations $X$ and $Y$. Furthermore, figure 18 shows that the quasinormal modes of higher excitations $n>1$ move to larger frequencies and closer to the real axis. This corresponds to the formation of stable massive mesons. Such a behavior is known in gauge/gravity duality for mesons which are built from massive quarks (e.g. [33]). Thus we observe a dynamical mass generation for the mesons as described in the following subsection. 


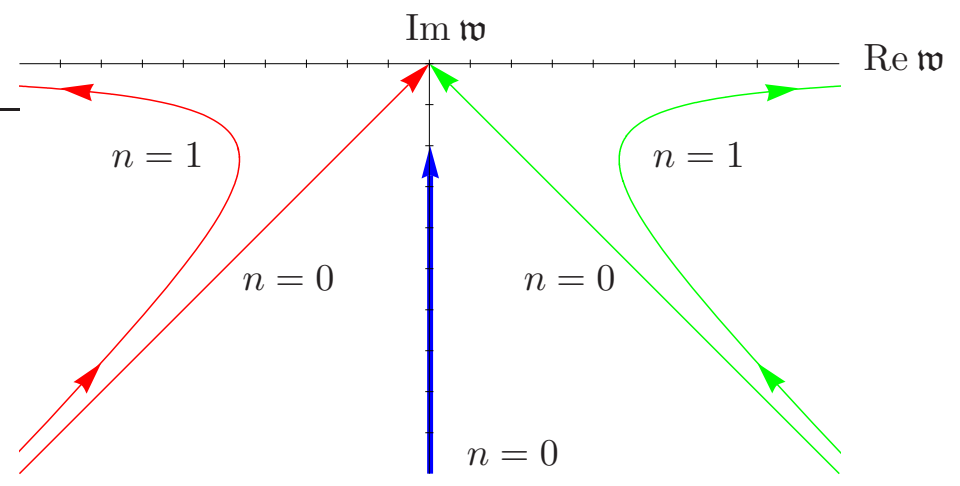

Figure 18. Movement of quasinormal modes under changes of the temperature $T$ : The different colors indicate the different fluctuations $X$ (red), $Y$ (green) and $a_{2}^{3}$ (blue). The higher excitations of the fields $X$ and $Y$ behave as excitations with a non-zero quark mass. This indicates a dynamical generation of the meson mass.

\subsubsection{Dynamical mass generation}

In this section we discuss dynamical mas generation on the field theory and on the gravity side. For this issue, it is important to distinguish between the cases where the broken symmetry is global or local in the boundary field theory.

Field theory observation. As explained in section 3.2, the superconducting condensate breaks the $\mathrm{U}(1)_{3}$ symmetry spontaneously in the field theory living on the AdS boundary. According to the Goldstone theorem this generates one massless Nambu-Goldstone boson in the boundary field theory. Let us now discuss the two different cases:

Local $\mathrm{U}(\mathbf{1})_{3}$ symmetry: if the broken symmetry is gauged, the Nambu-Goldstone boson is eaten by the gauge field $A^{3}$ charged under the spontaneously broken $\mathrm{U}(1)_{3}$ symmetry. In conventional superconductors this mechanism gives mass to the photons which implies the Meissner-Ochsenfeld effect.

Global $\mathrm{U}(\mathbf{1})_{3}$ symmetry: if the broken symmetry is global, there is no dynamical gauge field which eats the Nambu-Goldstone boson. Thus the Nambu-Goldstone boson remains present in the spectrum. Since this is the case in our setup, we need to identify this Nambu-Goldstone boson in the spectrum. In general, the Nambu-Goldstone boson corresponds to the phase of the condensate which parametrizes the the coset space $\mathrm{U}(1)_{3} / \mathbb{Z}_{2}$. In our setup, the fluctuations $X$ and $Y$ defined above (6.12) are charged under the $\mathrm{U}(1)_{3}$ symmetry such that the Nambu-Goldstone boson can be found in these fluctuations (see figure 18).

Although in our case the broken symmetry is global, we nevertheless observe dynamical mass generation as see for instance in figures 16 and 18. Therefore in our setup, a more subtle mechanism then the ordinary Higgs mechanism generates the meson masses dynamically. Let us explain this mechanism in the dual gravity setup. 
Gravity explanation. According to the AdS/CFT dictionary, a global symmetry in the boundary field theory is mapped to a local symmetry in the gravity theory. Thus the $\mathrm{SU}(2)$ symmetry of the two D7-branes is local in the gravity theory. In this paper we find new solutions to the equations of motion which non-vanishing gauge field components $A_{0}^{3}$ and $A_{3}^{1}$. Since the gravity action is unchanged and thus still $\mathrm{SU}(2)$ invariant, this non-zero components break the $\mathrm{SU}(2)$ gauge symmetry spontaneously which generates in total three Nambu-Goldstone bosons. They are eaten by the gauge fields which then become massive. Let us now discuss the corresponding mass terms in more detail.

First we consider a non-zero $\mathrm{SU}(2)$ isospin chemical potential $A_{0}^{3}$ which generates the mass term $\left(A_{0}^{3}\right)^{2}\left(a^{1,2}\right)^{2}$ for the fluctuations $a^{1,2}$. This new term shifts the quadratic fluctuation term in the action, i.e. its energy by the value of the field $A_{0}^{3}$. For the spectral function of the dual field theory this results in a shift of the meson resonance peaks, see [33].

Second we consider the non-zero gauge field component $A_{3}^{1}$ which generates a mass term $\left(A_{3}^{1}\right)^{2}\left(a^{3}\right)^{2}$ for the fluctuation $a^{3}$. We expect that this mass term shifts the quasinormal frequencies of this system in the complex plane. That results in generation and shift of the resonance peaks in the spectral function as well as generation of the gap structure in the conductivity. To make more precise statements, the spectrum of quasinormal modes needs to be studied in presence of these fields.

\section{Meissner-Ochsenfeld effect}

The Meissner effect is a distinct signature of conventional and unconventional superconductors. It is the phenomenon of expulsion of external magnetic fields. An induced current in the superconductor generates a magnetic field counter-acting the external magnetic field $H$. In AdS/CFT we are not able to observe the generation of counter-fields since the symmetries on the boundary are always global. Nevertheless, we can study their cause, i.e. the current induced in the superconductor. As usual $[4-6,9,13,18]$ the philosophy here is to weakly gauge the boundary theory afterwards.

In order to investigate how an external magnetic field influences our p-wave superconductor, we have two choices. Either we introduce the field along the spatial $z$-direction $H_{3}^{3} \tau^{3}$ or equivalently one along the $y$-direction i.e. $H_{2}^{3} \tau^{3}$. Both are "aligned" with the spontaneously broken $\mathrm{U}(1)_{3}$-flavor direction.

As an example here we choose a non-vanishing $H_{3}^{3} \tau^{3}$. This requires inclusion of some more non-vanishing field strength components in addition to those given in equation (3.9). In particular we choose $A_{0}^{3}(\varrho, x), A_{3}^{1}(\varrho, x)$ and $A_{2}^{3}(x)=x^{1} H_{3}^{3}$ yielding the additional components ${ }^{5}$

$$
\begin{aligned}
& H_{3}^{3}=F_{12}^{3}=-F_{21}^{3}=\partial_{1} A_{2}^{3}, \\
& F_{13}^{1}=-F_{31}^{1}=\partial_{1} A_{3}^{1}, \\
& F_{23}^{2}=-F_{32}^{2}=\frac{\gamma}{\sqrt{\lambda}} A_{2}^{3} A_{3}^{1}, \\
& F_{10}^{3}=-F_{01}^{3}=\partial_{1} A_{0}^{3} .
\end{aligned}
$$

\footnotetext{
${ }^{5}$ Close to the phase transition, it is consistent to drop the dependence of the field $H_{3}^{3}$ on $\varrho$. Away form the phase transition the $\varrho$ dependence must be included. From the boundary asymptotics it will be possible to extract the magnetic field and the magnetization of the superconductor.
} 
Recall that the radial AdS-direction is identified by the indices either $\varrho$ or 4. Amending the DBI-action (3.12) with the additional components (7.1), we compute the determinant in analogy to equation (3.12). We then choose to expand the new action to second order in $F$, i.e. we only consider terms being at most quadratic in the fields. This procedure gives the truncated DBI action

$$
\begin{aligned}
S_{\mathrm{DBI}}=-T_{D 7} N_{f} & \int \mathrm{d}^{8} \xi \sqrt{-G}\left[1+\frac{\left(2 \pi \alpha^{\prime}\right)^{2}}{2}\left(G^{00} G^{33}\left(F_{03}^{2}\right)^{2}+G^{33} G^{44}\left(F_{\varrho 3}^{1}\right)^{2}+G^{00} G^{44}\left(F_{\varrho 0}^{3}\right)^{2}\right.\right. \\
& \left.\left.+\left(G^{33}\right)^{2}\left(F_{13}^{1}\right)^{2}+\left(G^{33}\right)^{2}\left(F_{23}^{2}\right)^{2}+G^{00} G^{33}\left(F_{10}^{3}\right)^{2}+\left(G^{33}\right)^{2}\left(F_{12}^{3}\right)^{2}\right)+\cdots\right] .
\end{aligned}
$$

Respecting the symmetries and variable dependencies in our specific system, this can be written as

$$
\begin{gathered}
S_{\mathrm{DBI}}=-T_{D 7} N_{f} \int \mathrm{d}^{8} \xi \sqrt{-G}\left[1+\frac{1}{2}\left(G^{00} G^{33}\left(\partial_{\overline{1}} \tilde{A}_{0}^{3}\right)^{2}+G^{33} G^{44}\left(\partial_{\varrho} \tilde{A}_{3}^{1}\right)^{2}+G^{00} G^{44}\left(\partial_{\varrho} \tilde{A}_{0}^{3}\right)^{2}\right.\right. \\
+\left(G^{33}\right)^{2}\left(\partial_{\overline{1}} \tilde{A}_{3}^{1}\right)^{2}+\left(G^{33}\right)^{2}\left(\bar{H}_{3}^{3}\right)^{2}+G^{00} G^{33} \frac{\varrho_{H}^{4} \gamma^{2}}{\left(2 \pi \alpha^{\prime}\right)^{2} \lambda}\left(\tilde{A}_{0}^{3} \tilde{A}_{3}^{1}\right)^{2} \\
\left.\left.+\left(G^{33}\right)^{2} \frac{\varrho_{H}^{4} \gamma^{2}}{\left(2 \pi \alpha^{\prime}\right)^{2} \lambda}\left(\tilde{A}_{3}^{1} \bar{H}_{3}^{3}\right)^{2} \bar{x}^{2}\right)+\cdots\right]
\end{gathered}
$$

with the convenient redefinitions

$$
\tilde{A}=\frac{2 \pi \alpha^{\prime}}{\varrho_{H}} A, \quad x=\varrho_{H} \bar{x}, \quad \bar{H}_{3}^{3}=2 \pi \alpha^{\prime} H_{3}^{3}, \quad \varrho=\varrho_{H} \rho .
$$

Rescaling the $\bar{x}$-coordinate once more

$$
\tilde{x}=\sqrt{\frac{\bar{H}_{3}^{3} \varrho^{2} \gamma}{2 \pi \alpha^{\prime} \sqrt{\lambda}}} \bar{x},
$$

the equations of motion derived from the action (7.3) take a simple form

$$
\begin{aligned}
& 0=\partial_{\rho}^{2} \tilde{A}_{0}^{3}+\frac{\partial_{\rho}\left[\sqrt{-G} G^{00} G^{44}\right]}{\sqrt{-G} G^{00} G^{44}} \partial_{\rho} \tilde{A}_{0}^{3}+\frac{\gamma \tilde{H}_{3}^{3}}{2 \sqrt{2} \pi} \frac{G^{33}}{G^{44}} \partial_{\tilde{x}} \tilde{A}_{0}^{3}-\frac{\gamma^{2}}{2 \pi^{2}} \frac{G^{33}}{G^{44}} \tilde{A}_{0}^{3}\left(\tilde{A}_{3}^{1}\right)^{2} \\
& 0=\partial_{\rho}^{2} \tilde{A}_{3}^{1}+\frac{\partial_{\rho}\left[\sqrt{-G} G^{33} G^{44}\right]}{\sqrt{-G} G^{33} G^{44}} \partial_{\rho} \tilde{A}_{3}^{1}+\frac{\gamma \tilde{H}_{3}^{3}}{2 \sqrt{2} \pi} \frac{G^{33}}{G^{44}}\left[\partial_{\tilde{x}}^{2} \tilde{A}_{3}^{1}-\tilde{x}^{2} \tilde{A}_{3}^{1}\right]-\frac{\gamma^{2}}{2 \pi^{2}} \frac{G^{00}}{G^{44}}\left(\tilde{A}_{0}^{3}\right)^{2} \tilde{A}_{3}^{1} .
\end{aligned}
$$

Here all metric components are understood to be evaluated at $R=1$ and $\varrho \rightarrow \rho$.

We aim at decoupling and solving the system of partial differential equations (7.6) by the product ansatz

$$
\tilde{A}_{3}^{1}(\rho, \tilde{x})=\mathfrak{g}(\rho) \mathfrak{f}(\tilde{x}) .
$$

For this ansatz to work, we need to make two assumptions: First we assume that $\tilde{A}_{0}^{3}$ is constant in $\tilde{x}$. Second we assume that $A_{3}^{1}$ is small, which clearly is the case near the 
transition $T \rightarrow T_{c}$. Our second assumption prevents $A_{0}^{3}$ from receiving a dependence on $\tilde{x}$ through its coupling to $A_{3}^{1}(\rho, \tilde{x})$. These assumptions allow to write the second equation in $(7.6)$ as

$$
\begin{aligned}
0=\partial_{\rho}^{2} \mathfrak{g}(\rho)+\frac{\partial_{\rho}\left[\sqrt{-G} G^{33} G^{44}\right]}{\sqrt{-G} G^{33} G^{44}} \partial_{\rho} \mathfrak{g} & (\rho)-\frac{\gamma^{2}}{2 \pi^{2}} \frac{G^{00}}{G^{44}}\left(\tilde{A}_{0}^{3}\right)^{2} \mathfrak{g}(\rho) \\
& +\frac{\gamma \tilde{H}_{3}^{3}}{2 \sqrt{2} \pi} \frac{G^{33}}{G^{44}} \mathfrak{g}(\rho) \frac{\partial_{\tilde{x}}^{2} \mathfrak{f}(\tilde{x})-\tilde{x}^{2} \mathfrak{f}(\tilde{x})}{\mathfrak{f}(\tilde{x})} .
\end{aligned}
$$

All terms but the last one are independent of $\tilde{x}$, so the product ansatz (7.7) is consistent only if

$$
\frac{\partial_{\tilde{x}}^{2} \mathfrak{f}(\tilde{x})-\tilde{x}^{2} \mathfrak{f}(\tilde{x})}{\mathfrak{f}(\tilde{x})}=C,
$$

where $C$ is a constant. The differential equation (7.9) has a particular solution if $C=$ $-(2 n+1), n \in \mathbb{N}$. The solutions for $\mathfrak{f}(\tilde{x})$ are Hermite functions

$$
\mathfrak{f}_{n}(\tilde{x})=\frac{e^{-\frac{|\tilde{x}|^{2}}{2}}}{\sqrt{n ! 2^{n} \sqrt{\pi}}} H_{n}(\tilde{x}), H_{n}(\tilde{x})=(-1)^{n} e^{\frac{|\tilde{x}|^{2}}{2}} \frac{d^{n}}{d x^{n}} e^{-\frac{|\tilde{x}|^{2}}{2}},
$$

which have Gaussian decay at large $|\tilde{x}| \gg 1$. Choosing the lowest solution with $n=0$ and $H_{0}=1$, which has no nodes, is most likely to give the configuration with lowest energy content. So the system we need to solve is finally given by

$$
\begin{aligned}
& 0=\partial_{\rho}^{2} \tilde{A}_{0}^{3}+\frac{\partial_{\rho}\left[\sqrt{-G} G^{00} G^{44}\right]}{\sqrt{-G} G^{00} G^{44}} \partial_{\rho} \tilde{A}_{0}^{3} \frac{\gamma^{2}}{2 \pi^{2}} \frac{G^{33}}{G^{44}} \tilde{A}_{0}^{3}\left(\mathfrak{f}_{0}(\tilde{x}) \mathfrak{g}(\rho)\right)^{2}, \\
& 0=\partial_{\rho}^{2} \mathfrak{g}+\frac{\partial_{\rho}\left[\sqrt{-G} G^{33} G^{44}\right]}{\sqrt{-G} G^{33} G^{44}} \partial_{\rho} \mathfrak{g}-\frac{\gamma^{2}}{2 \pi^{2}} \frac{G^{00}}{G^{44}}\left(\tilde{A}_{0}^{3}\right)^{2} \mathfrak{g}-\frac{\gamma \tilde{H}_{3}^{3}}{2 \sqrt{2} \pi} \frac{G^{33}}{G^{44}} \mathfrak{g} .
\end{aligned}
$$

Asymptotically near the horizon the fields take the form

$$
\begin{array}{llll}
\tilde{A}_{0}^{3}= & +a_{2}(\rho-1)^{2} & +\mathcal{O}\left((\rho-1)^{3}\right), \\
\mathfrak{g} & =b_{0} & +\frac{b_{0} H_{3}^{3}}{4}(\rho-1)^{2} & +\mathcal{O}\left((\rho-1)^{-3}\right),
\end{array}
$$

while at the boundary we obtain

$$
\begin{aligned}
& \tilde{A}_{0}^{3}=\tilde{\mu} \quad+\frac{\tilde{d}_{0}^{3}}{\rho^{2}}+\mathcal{O}\left(\rho^{-4}\right), \\
& \mathfrak{g}=\quad+\frac{\tilde{d}_{3}^{1}}{\rho^{2}} \quad+\mathcal{O}\left(\rho^{-4}\right) .
\end{aligned}
$$

We succeed in finding numerical solutions $\mathfrak{g}(\rho)$ and $A_{0}^{3}(\rho)$ to the set of equations (7.11) obeying the asymptotics given by equations (7.12) and (7.13). These numerical solutions are used to approach the phase transition from the superconducting phase by increasing the magnetic field. We map out the line of critical temperature-magnetic field pairs in figure 19. In this way we obtain a phase diagram displaying the Meissner effect. The critical line in figure 19 separates the phase with and without superconducting condensate $\tilde{d}_{3}^{1}$. 


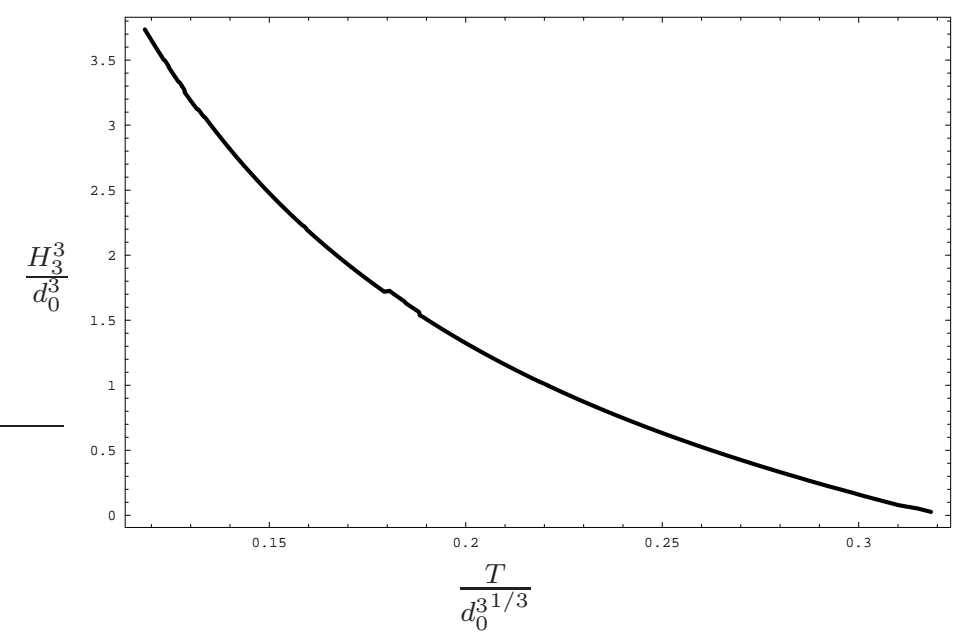

Figure 19. The line of critical magnetic field versus critical temperature. Below this line the external magnetic field coexists with the superconducting condensate. Above the line the superconducting condensate vanishes. We set the spatial position arbitrarily to $\tilde{x}=0.1$ since the critical line does not depend on $\tilde{x}$.

We emphasize that this is a background calculation involving no fluctuations. Complementary to the procedure described above we also confirmed the phase diagram using the instability of the normal phase against fluctuations. Starting at large magnetic field and vanishing condensate $\tilde{d}_{3}^{1}$, we determine for a given magnetic field $H_{3}^{3}$ the temperature $T_{c}\left(H_{3}^{3}\right)$ at which the fluctuation $a_{1}^{3}$ becomes unstable. That instability signals the condensation process into the superconducting phase.

The presence of the coexistence phase below the critical line, where the system is still superconducting despite the presence of an external magnetic field, is the signal of the Meissner effect in the case of a global symmetry considered here. If we now weakly gauged the flavor symmetry at the boundary, the superconducting current $J_{3}^{1}$ would generate a magnetic field opposite to the external field. Thus the phase observed is a necessary condition in the case of a global symmetry for finding the standard Meissner effect when gauging the symmetry.

\section{Discussion and outlook}

We found a holographic realization of superconductivity in the context of gauge/gravity duality with flavor at finite isospin chemical potential, for which the field theory action is known explicitly. The condensation process corresponds to a recombination of strings which leads to a thermodynamically favored configuration. On the field theory side, this new state may be interpreted as a $\rho$ meson superfluid. We show that the superconducting phase persists at finite quark mass and find a holographic Meissner-Ochsenfeld effect. From a technical point of view, we compare two different approaches for evaluating the nonAbelian DBI action involved (expansion to fourth order and a new modified trace evaluation procedure which we propose) and find the same physical results in both approaches, at least qualitatively. 
For the future, we expect that a holographic s-wave superconductor may be constructed by applying the methods presented to the transverse scalars in similar holographic setups. It will also be interesting to apply our methods to the configuration discussed in [48] in order to find a purely fermionic superconductor.

\section{Acknowledgments}

We are grateful to T. Dahm, S. Gubser, S. Hartnoll, C. Herzog, K. Landsteiner, R. Meyer, A. O'Bannon, S. Pufu, F. Rust and E. Tsatis for discussions. This work was supported in part by The Cluster of Excellence for Fundamental Physics - Origin and Structure of the Universe.

\section{A Expansion of the DBI action to fourth order}

In this section we give some lengthy equations needed to calculate the expanded DBI action and the resulting equations of motion (see section 3.2.2).

The $\mathcal{T}_{i}$ which determine the terms in expanded DBI action at $i-t h$ order in the field strength are given by

$$
\begin{gathered}
\mathcal{T}_{2}=\left(2 \pi \alpha^{\prime}\right)^{2}\left[G^{00} G^{44}\left(F_{\varrho 0}^{3}\right)^{2}+G^{33} G^{44}\left(F_{\varrho 3}^{1}\right)^{2}+G^{00} G^{33}\left(F_{03}^{2}\right)^{2}\right] \\
=-\frac{2 \tilde{f} H}{f^{2}}\left(\partial_{\rho} \tilde{A}_{0}^{3}\right)^{2}+\frac{2 H}{\tilde{f}}\left(\partial_{\rho} \tilde{A}_{3}^{1}\right)^{2}-\frac{2 \gamma^{2}}{\rho^{4} f^{2} \pi^{2}}\left(\tilde{A}_{0}^{3} \tilde{A}_{3}^{1}\right)^{2} \\
\mathcal{T}_{4}=\left(2 \pi \alpha^{\prime}\right)^{4}\left[\left(G^{00} G^{44}\right)^{2}\left(F_{\varrho 0}^{3}\right)^{4}+\left(G^{33} G^{44}\right)^{2}\left(F_{\varrho 3}^{1}\right)^{4}+\left(G^{00} G^{33}\right)^{2}\left(F_{03}^{2}\right)^{4}\right. \\
+\frac{2}{3} G^{00} G^{33}\left(G^{44}\right)^{2}\left(F_{\varrho 0}^{3}\right)^{2}\left(F_{\varrho 3}^{1}\right)^{2}+\frac{2}{3}\left(G^{00}\right)^{2} G^{33} G^{44}\left(F_{\varrho 0}^{3}\right)^{2}\left(F_{03}^{2}\right)^{2} \\
\left.+\frac{2}{3} G^{00}\left(G^{33}\right)^{2} G^{44}\left(F_{\varrho 3}^{1}\right)^{2}\left(F_{03}^{2}\right)^{2}\right] \\
=\frac{4 \tilde{f}^{2} H^{2}}{f^{4}}\left(\partial_{\rho} \tilde{A}_{0}^{3}\right)^{4}+\frac{4 H^{2}}{\tilde{f}^{2}}\left(\partial_{\rho} \tilde{A}_{3}^{1}\right)^{4}+\frac{4 \gamma^{4}}{\rho^{8} f^{4} \pi^{4}}\left(\tilde{A}_{0}^{3} \tilde{A}_{3}^{1}\right)^{4}-\frac{8 H^{2}}{3 f^{2}}\left(\partial_{\rho} \tilde{A}_{0}^{3}\right)^{2}\left(\partial_{\rho} \tilde{A}_{3}^{1}\right)^{2} \\
+\frac{8 \tilde{f} H \gamma^{2}}{3 \rho^{4} f^{4} \pi^{2}}\left(\partial_{\rho} \tilde{A}_{0}^{3}\right)^{2}\left(\tilde{A}_{0}^{3} \tilde{A}_{3}^{1}\right)^{2}-\frac{8 H \gamma^{2}}{3 \rho^{4} \tilde{f} f^{2} \pi^{2}}\left(\partial_{\rho} \tilde{A}_{3}^{1}\right)^{2}\left(\tilde{A}_{0}^{3} \tilde{A}_{3}^{1}\right)^{2},
\end{gathered}
$$

with

$$
H=\frac{1-\chi^{2}}{1-\chi^{2}+\rho^{2}\left(\partial_{\rho} \chi\right)^{2}}
$$


Using the Euler-Lagrange equation the resulting equations of motion are

$$
\begin{aligned}
& \partial_{\rho}\left[\sqrt{-G}\left(\frac{2 \tilde{f} H}{f^{2}}\left(\partial_{\rho} \tilde{A}_{0}^{3}+\frac{2 \tilde{f}^{2} H^{2}}{f^{4}}\left(\partial_{\rho} \tilde{A}_{0}^{3}\right)^{3}-\frac{2 H^{2}}{3 f^{2}}\left(\partial_{\rho} \tilde{A}_{3}^{1}\right)^{2}\left(\partial_{\rho} \tilde{A}_{0}^{3}\right)+\frac{2 \tilde{f} \gamma^{2} H}{3 f^{4} \rho^{4} \pi^{2}}\left(\partial_{\rho} \tilde{A}_{0}^{3}\right)\left(\tilde{A}_{0}^{3} \tilde{A}_{3}^{1}\right)^{2}\right)\right]\right. \\
& =\frac{2 \gamma^{2} \sqrt{-G}}{\pi^{2} \rho^{4} f^{2}}\left[\tilde{A}_{0}^{3}\left(\tilde{A}_{3}^{1}\right)^{2}+\frac{\gamma^{2}}{\pi^{2} \rho^{4} f^{2}}\left(\tilde{A}_{0}^{3}\right)^{3}\left(\tilde{A}_{3}^{1}\right)^{4}+\frac{\tilde{f} H}{3 f^{2}}\left(\partial_{\rho} \tilde{A}_{0}^{3}\right)^{2} \tilde{A}_{0}^{3}\left(\tilde{A}_{3}^{1}\right)^{2}-\frac{H}{3 \tilde{f}}\left(\partial_{\rho} \tilde{A}_{3}^{1}\right)^{2} \tilde{A}_{0}^{3}\left(\tilde{A}_{3}^{1}\right)^{2}\right], \\
& \partial_{\rho}\left[\sqrt{-G}\left(\frac{2 H}{\tilde{f}}\left(\partial_{\rho} \tilde{A}_{3}^{1}\right)-\frac{2 H^{2}}{\tilde{f}^{2}}\left(\partial_{\rho} \tilde{A}_{3}^{1}\right)^{3}+\frac{2 H^{2}}{3 f^{2}}\left(\partial_{\rho} \tilde{A}_{0}^{3}\right)^{2}\left(\partial_{\rho} \tilde{A}_{3}^{1}\right)+\frac{2 \gamma^{2} H}{3 \pi^{2} \rho^{4} f^{2} \tilde{f}}\left(\partial_{\rho} \tilde{A}_{3}^{1}\right)\left(\tilde{A}_{0}^{3} \tilde{A}_{3}^{1}\right)^{2}\right)\right] \\
& =-\frac{2 \gamma^{2} \sqrt{-G}}{\pi^{2} \rho^{4} f^{2}}\left[\left(\tilde{A}_{0}^{3}\right)^{2} \tilde{A}_{3}^{1}+\frac{\gamma^{2}}{\pi^{2} \rho^{4} f^{2}}\left(\tilde{A}_{0}^{3}\right)^{4}\left(\tilde{A}_{3}^{1}\right)^{3}+\frac{\tilde{f} H}{3 f^{2}}\left(\partial_{\rho} \tilde{A}_{0}^{3}\right)^{2}\left(\tilde{A}_{0}^{3}\right)^{2} \tilde{A}_{3}^{1}-\frac{H}{3 \tilde{f}}\left(\partial_{\rho} \tilde{A}_{3}^{1}\right)^{2}\left(\tilde{A}_{0}^{3}\right)^{2} \tilde{A}_{3}^{1}\right], \\
& \partial_{\rho}\left[\frac{\rho^{5} f \tilde{f}\left(1-\chi^{2}\right)\left(\partial_{\rho} \chi\right)}{\sqrt{1-\chi^{2}+\rho^{2}\left(\partial_{\rho} \chi\right)^{2}}}\left(1+\frac{\mathcal{T}_{2}}{2}-\frac{\mathcal{T}_{4}}{8}-\frac{\varrho_{H}^{2}}{R^{2}} \frac{2 \rho^{2}\left(1-\chi^{2}\right)}{1-\rho^{2}\left(\partial_{\rho} \chi\right)^{2}}\left[\frac{1}{2} \frac{\partial \mathcal{T}_{2}}{\partial G^{44}}-\frac{1}{8} \frac{\partial \mathcal{T}_{4}}{\partial G^{44}}\right]\right)\right] \\
& =-\frac{\rho^{3} f \tilde{f} \chi}{\sqrt{1-\chi^{2}+\rho^{2}\left(\partial_{\rho} \chi\right)^{2}}}\left[\left(3\left(1-\chi^{2}\right)+2 \rho^{2}\left(\partial_{\rho} \chi\right)^{2}\right)\left(1+\frac{\mathcal{T}_{2}}{2}-\frac{\mathcal{T}_{4}}{8}\right)\right. \\
& \left.+\frac{\varrho_{H}^{2}}{R^{2}} \frac{2 \rho^{4}\left(1-\chi^{2}\right)\left(\partial_{\rho} \chi\right)^{2}}{1-\chi^{2}+\rho^{2}\left(\partial_{\rho} \chi\right)^{2}}\left(\frac{1}{2} \frac{\partial \mathcal{T}_{2}}{\partial G^{44}}-\frac{1}{8} \frac{\partial \mathcal{T}_{4}}{\partial G^{44}}\right)\right],
\end{aligned}
$$

with

$$
\begin{aligned}
& \frac{\partial \mathcal{T}_{2}}{\partial G^{44}}=\frac{2 R^{2}}{\varrho_{H}^{2} \rho^{2}} {\left[-\frac{\tilde{f}}{f^{2}}\left(\partial_{\rho} \tilde{A}_{0}^{3}\right)^{2}+\frac{1}{\tilde{f}}\left(\partial_{\rho} \tilde{A}_{3}^{1}\right)^{2}\right], } \\
& \frac{\partial \mathcal{T}_{4}}{\partial G^{44}}=\frac{2 R^{2}}{\varrho_{H}^{2} \rho^{2}}\left[\frac{4 \tilde{f}^{2} H}{f^{4}}\left(\partial_{\rho} \tilde{A}_{0}^{3}\right)^{4}+\frac{4 H}{\tilde{f}^{2}}\left(\partial_{\rho} \tilde{A}_{3}^{1}\right)^{4}-\frac{8 H}{3 f^{2}}\left(\partial_{\rho} \tilde{A}_{0}^{3}\right)^{2}\left(\partial_{\rho} \tilde{A}_{3}^{1}\right)^{2}\right. \\
&\left.\quad+\frac{4 \gamma^{2} \tilde{f}}{3 \rho^{4} f^{4} \pi^{2}}\left(\partial_{\rho} \tilde{A}_{0}^{3}\right)^{2}\left(\tilde{A}_{0}^{3} \tilde{A}_{3}^{1}\right)^{2}-\frac{4 \gamma^{2}}{3 \rho^{4} \tilde{f} f^{2} \pi^{2}}\left(\partial_{\rho} \tilde{A}_{3}^{1}\right)^{2}\left(\tilde{A}_{0}^{3} \tilde{A}_{3}^{1}\right)^{2}\right] .
\end{aligned}
$$

\section{B Fluctuations}

\section{B.1 Adapted symmetrized trace prescription}

In this section we present the elements of the inverse background tensor $\mathcal{G}^{\mu \nu}$ defined in section 6.1. The diagonal elements are given by

$$
\begin{aligned}
\mathcal{G}^{00} & =G^{00} \frac{1+G^{33} G^{44}\left(\partial_{\rho} \tilde{A}_{3}^{1}\right)^{2}}{N}, \\
\mathcal{G}^{33} & =G^{33} \frac{1+G^{00} G^{44}\left(\partial_{\rho} \tilde{A}_{0}^{3}\right)^{2}}{N}, \\
\mathcal{G}^{44} & =G^{44} \frac{1-\frac{2 \gamma^{2}}{\pi^{2} \rho^{4} f^{2}}\left(\tilde{A}_{3}^{1} \tilde{A}_{0}^{3}\right)^{2}}{N},
\end{aligned}
$$


where the other diagonal elements are just $\mathcal{G}^{i i}=G^{i i}$. The off-diagonal elements read

$$
\begin{aligned}
\mathcal{G}^{34} & =G^{44} \frac{G^{33}\left(\partial_{\rho} \tilde{A}_{3}^{1}\right) \tau^{1}-\frac{2 \sqrt{2} \gamma}{\pi \rho^{4} f^{2}} \frac{R^{2}}{\varrho_{H}^{2}} \tilde{A}_{3}^{1} \tilde{A}_{0}^{3}\left(\partial_{\rho} \tilde{A}_{0}^{3}\right) \tau^{2} \tau^{3}}{N}, \\
\mathcal{G}^{43} & =G^{44} \frac{-G^{33}\left(\partial_{\rho} \tilde{A}_{3}^{1}\right) \tau^{1}-\frac{2 \sqrt{2} \gamma}{\pi \rho^{4} f^{2}} \frac{R}{\varrho}_{H}^{2}}{N} \tilde{A}_{3}^{1} \tilde{A}_{0}^{3}\left(\partial_{\rho} \tilde{A}_{0}^{3}\right) \tau^{2} \tau^{3} \\
\mathcal{G}^{03} & =\frac{4 R^{2}}{\varrho_{H}^{2} \rho^{4} f^{2}} \frac{\frac{\sqrt{2} \gamma}{2 \pi} \tilde{A}_{3}^{1} \tilde{A}_{0}^{3} \tau^{2}+\frac{R^{2}}{\varrho_{H}^{2}} G^{44}\left(\partial_{\rho} \tilde{A}_{3}^{1}\right)\left(\partial_{\rho} \tilde{A}_{0}^{3}\right) \tau^{1} \tau^{3}}{N}, \\
\mathcal{G}^{30} & =\frac{4 R^{2}}{\varrho_{H}^{2} \rho^{4} f^{2}} \frac{-\frac{\sqrt{2} \gamma}{2 \pi} \tilde{A}_{3}^{1} \tilde{A}_{0}^{3} \tau^{2}+\frac{R^{2}}{\varrho_{H}^{2}} G^{44}\left(\partial_{\rho} \tilde{A}_{3}^{1}\right)\left(\partial_{\rho} \tilde{A}_{0}^{3}\right) \tau^{1} \tau^{3}}{N} \\
\mathcal{G}^{04} & =G^{00} G^{44} \frac{\left(\partial_{\rho} \tilde{A}_{0}^{3}\right) \tau^{3}-\frac{\sqrt{2} \gamma}{2 \pi} \frac{\varrho_{H}^{2}}{R^{2}} G^{33} \tilde{A}_{3}^{1} \tilde{A}_{0}^{3}\left(\partial_{\rho} \tilde{A}_{3}^{1}\right) \tau^{1} \tau^{2}}{N} \\
\mathcal{G}^{40} & =G^{00} G^{44} \frac{-\left(\partial_{\rho} \tilde{A}_{0}^{3}\right) \tau^{3}-\frac{\sqrt{2} \gamma}{2 \pi} \frac{\varrho_{H}^{2}}{R^{2}} G^{33} \tilde{A}_{3}^{1} \tilde{A}_{0}^{3}\left(\partial_{\rho} \tilde{A}_{3}^{1}\right) \tau^{1} \tau^{2}}{N} .
\end{aligned}
$$

The denominator $N$ is given by

$$
N=1+G^{33} G^{44}\left(\partial_{\rho} \tilde{A}_{3}^{1}\right)^{2}+G^{00} G^{44}\left(\partial_{\rho} \tilde{A}_{0}^{3}\right)^{2}-\frac{2 \gamma^{2}}{\pi^{2} \rho^{4} f^{2}}\left(\tilde{A}_{3}^{1} \tilde{A}_{0}^{3}\right)^{2}
$$

\section{B.2 Expansion of the DBI action to fourth order}

In this section we calculate the quadratic action for the fluctuations using the expanded DBI action as explained in section 6.2. Since the fluctuation $a_{2}^{3}$ decouples from every other fluctuation we can write down an effective Lagrangian for this fluctuation. This Lagrangian is given by

$$
\begin{aligned}
\mathcal{L}_{\text {eff }}^{a_{2}^{3}} & =\sqrt{-G} \operatorname{Str}\left[\frac{1}{2} G^{22} G^{44}\left(\check{F}_{24}^{3}\right)^{2}\left(\sigma^{3}\right)^{2}+\frac{1}{2} G^{00} G^{22}\left(\check{F}_{02}^{3}\right)^{2}\left(\sigma^{3}\right)^{2}+\frac{1}{2} G^{22} G^{33}\left(\check{F}_{23}^{2}\right)^{2}\left(\sigma^{2}\right)^{2}\right. \\
& +\frac{1}{4} G^{33} G^{44}\left(F_{34}^{1}\right)^{2}\left[G^{00} G^{22}\left(\check{F}_{02}^{3}\right)^{2}\left(\sigma^{3} \sigma^{1}\right)^{2}-G^{22} G^{44}\left(\check{F}_{24}^{3}\right)^{2}\left(\sigma^{3} \sigma^{1}\right)^{2}-G^{22} G^{33}\left(\check{F}_{23}^{2}\right)^{2}\left(\sigma^{2} \sigma^{1}\right)^{2}\right] \\
& +\frac{1}{4} G^{00} G^{33}\left(F_{03}^{2}\right)^{2}\left[G^{22} G^{44}\left(\check{F}_{24}^{3}\right)^{2}\left(\sigma^{3} \sigma^{2}\right)^{2}-G^{00} G^{22}\left(\check{F}_{02}^{3}\right)^{2}\left(\sigma^{3} \sigma^{2}\right)^{2}-G^{22} G^{33}\left(\check{F}_{23}^{2}\right)^{2}\left(\sigma^{2}\right)^{4}\right] \\
& +\frac{1}{4} G^{00} G^{44}\left(F_{04}^{3}\right)^{2}\left[G^{22} G^{33}\left(\check{F}_{23}^{2}\right)^{2}\left(\sigma^{3} \sigma^{2}\right)^{2}-G^{00} G^{22}\left(\check{F}_{02}^{3}\right)^{2}\left(\sigma^{3}\right)^{4}-G^{22} G^{44}\left(\check{F}_{24}^{3}\right)^{2}\left(\sigma^{3}\right)^{4}\right] \\
& +G^{00} G^{22} G^{33} G^{44}\left[\left(F_{34}^{1}\right)\left(F_{04}^{3}\right)\left(\check{F}_{02}^{3}\right)\left(\check{F}_{23}^{2}\right) \sigma^{1} \sigma^{2}\left(\sigma^{3}\right)^{2}-\left(F_{03}^{2}\right)\left(F_{04}^{3}\right)\left(\check{F}_{24}^{3}\right)\left(\check{F}_{23}^{2}\right)\left(\sigma^{2} \sigma^{3}\right)^{2}\right] \\
& \left.-G^{00} G^{22} G^{33} G^{44}\left(F_{03}^{2}\right)\left(F_{34}^{1}\right)\left(\check{F}_{02}^{3}\right)\left(\check{F}_{24}^{3}\right) \sigma^{1} \sigma^{2}\left(\sigma^{3}\right)^{2}\right] .
\end{aligned}
$$


Using the results for the symmetrized traces (3.28), the effective Lagrangian simplifies to

$$
\begin{aligned}
\mathcal{L}_{\text {eff }}^{a_{2}^{3}}= & \sqrt{-G}\left[\frac{1}{2} G^{22} G^{44}\left(\check{F}_{24}\right)^{2}+\frac{1}{2} G^{00} G^{22}\left(\check{F}_{02}^{3}\right)^{2}+\frac{1}{2} G^{22} G^{33}\left(\check{F}_{23}^{2}\right)^{2}\right. \\
& +\frac{1}{6} G^{33} G^{44}\left(F_{34}^{1}\right)^{2}\left[G^{00} G^{22}\left(\check{F}_{02}^{3}\right)^{2}-G^{22} G^{44}\left(\check{F}_{24}^{3}\right)^{2}-G^{22} G^{33}\left(\check{F}_{23}^{2}\right)^{2}\right] \\
& +\frac{1}{2} G^{00} G^{44}\left(F_{04}^{3}\right)^{2}\left[\frac{1}{3} G^{22} G^{33}\left(\check{F}_{23}^{2}\right)^{2}-\frac{1}{2} G^{22} G^{44}\left(\check{F}_{24}^{3}\right)^{2}-\frac{1}{2} G^{00} G^{22}\left(\check{F}_{02}^{3}\right)^{2}\right] \\
& +\frac{1}{2} G^{00} G^{33}\left(F_{03}^{2}\right)^{2}\left[-\frac{1}{3} G^{00} G^{22}\left(\check{F}_{02}^{3}\right)^{2}-\frac{1}{2} G^{22} G^{33}\left(\check{F}_{23}^{2}\right)^{2}+\frac{1}{3} G^{22} G^{44}\left(\check{F}_{24}^{3}\right)^{2}\right] \\
& \left.-\frac{2}{3} G^{00} G^{22} G^{33} G^{44}\left(F_{03}^{2}\right)\left(F_{04}^{2}\right)\left(\check{F}_{24}^{3}\right)\left(\check{F}_{23}^{2}\right)\right] .
\end{aligned}
$$

To simplify the equation of motion for the fluctuation $a_{2}^{3}$ (see (6.15)) we define

$$
\begin{aligned}
& \mathcal{H}^{00}=G^{00}\left[1+\frac{1}{3} G^{33} G^{44}\left(\partial_{\rho} \tilde{A}_{3}^{1}\right)^{2}-\frac{1}{2} G^{00} G^{44}\left(\partial_{\rho} \tilde{A}_{0}^{3}\right)^{2}-\frac{1}{3} \frac{\varrho_{H}^{4}}{R^{4}} G^{00} G^{33}\left(\frac{\gamma}{\sqrt{2}} \tilde{A}_{3}^{1} \tilde{A}_{0}^{3}\right)^{2}\right], \\
& \mathcal{H}^{33}=G^{33}\left[1-\frac{1}{3} G^{33} G^{44}\left(\partial_{\rho} \tilde{A}_{3}^{1}\right)^{2}-\frac{1}{3} G^{00} G^{44}\left(\partial_{\rho} \tilde{A}_{0}^{3}\right)^{2}-\frac{1}{2} \frac{\varrho_{H}^{4}}{R^{4}} G^{00} G^{33}\left(\frac{\gamma}{\sqrt{2}} \tilde{A}_{3}^{1} \tilde{A}_{0}^{3}\right)^{2}\right], \\
& \mathcal{H}^{44}=G^{44}\left[1-\frac{1}{3} G^{33} G^{44}\left(\partial_{\rho} \tilde{A}_{3}^{1}\right)^{2}-\frac{1}{2} G^{00} G^{44}\left(\partial_{\rho} \tilde{A}_{0}^{3}\right)^{2}+\frac{1}{3} \frac{\varrho_{H}^{4}}{R^{4}} G^{00} G^{33}\left(\frac{\gamma}{\sqrt{2}} \tilde{A}_{3}^{1} \tilde{A}_{0}^{3}\right)^{2}\right] .
\end{aligned}
$$

\section{References}

[1] J.M. Maldacena, The large- $N$ limit of superconformal field theories and supergravity, Adv. Theor. Math. Phys. 2 (1998) 231 [Int. J. Theor. Phys. 38 (1999) 1113] [hep-th/9711200] [SPIRES].

[2] E. Witten, Anti-de Sitter space, thermal phase transition and confinement in gauge theories, Adv. Theor. Math. Phys. 2 (1998) 505 [hep-th/9803131] [SPIRES].

[3] S.A. Hartnoll, C.P. Herzog and G.T. Horowitz, Building a holographic superconductor, Phys. Rev. Lett. 101 (2008) 031601 [arXiv:0803.3295] [SPIRES].

[4] E. Nakano and W.-Y. Wen, Critical magnetic field in a holographic superconductor, Phys. Rev. D 78 (2008) 046004 [arXiv:0804.3180] [SPIRES].

[5] T. Albash and C.V. Johnson, A holographic superconductor in an external magnetic field, JHEP 09 (2008) 121 [arXiv:0804.3466] [SPIRES].

[6] W.-Y. Wen, Inhomogeneous magnetic field in AdS/CFT superconductor, arXiv:0805.1550 [SPIRES].

[7] S.S. Gubser and S.S. Pufu, The gravity dual of a p-wave superconductor, JHEP 11 (2008) 033 [arXiv:0805.2960] [SPIRES].

[8] M.M. Roberts and S.A. Hartnoll, Pseudogap and time reversal breaking in a holographic superconductor, JHEP 08 (2008) 035 [arXiv:0805.3898] [SPIRES].

[9] K. Maeda and T. Okamura, Characteristic length of an AdS/CFT superconductor, Phys. Rev. D 78 (2008) 106006 [arXiv:0809.3079] [SPIRES]. 
[10] C.P. Herzog, P.K. Kovtun and D.T. Son, Holographic model of superfluidity, arXiv:0809. 4870 [SPIRES].

[11] P. Basu, A. Mukherjee and H.-H. Shieh, Supercurrent: vector hair for an AdS black hole, Phys. Rev. D 79 (2009) 045010 [arXiv:0809.4494] [SPIRES].

[12] G.T. Horowitz and M.M. Roberts, Holographic superconductors with various condensates, Phys. Rev. D 78 (2008) 126008 [arXiv:0810.1077] [SPIRES].

[13] S.A. Hartnoll, C.P. Herzog and G.T. Horowitz, Holographic superconductors, JHEP 12 (2008) 015 [arXiv: 0810.1563] [SPIRES].

[14] M. Ammon, J. Erdmenger, M. Kaminski and P. Kerner, Superconductivity from gauge/gravity duality with flavor, Phys. Lett. B 680 (2009) 516 [arXiv:0810.2316] [SPIRES].

[15] P. Basu, J. He, A. Mukherjee and H.-H. Shieh, Superconductivity from D3/D7: holographic pion superfluid, arXiv:0810.3970 [SPIRES].

[16] S.S. Gubser and A. Nellore, Low-temperature behavior of the Abelian Higgs model in Anti-de Sitter space, JHEP 04 (2009) 008 [arXiv: 0810.4554] [SPIRES].

[17] A. O'Bannon, Toward a holographic model of superconducting fermions, JHEP 01 (2009) 074 [arXiv:0811.0198] [SPIRES].

[18] A. Rebhan, A. Schmitt and S.A. Stricker, Meson supercurrents and the Meissner effect in the Sakai-Sugimoto model, JHEP 05 (2009) 084 [arXiv:0811.3533] [SPIRES].

[19] N. Evans and E. Threlfall, Chemical potential in the gravity dual of a $2+1$ dimensional system, Phys. Rev. D 79 (2009) 066008 [arXiv:0812.3273] [SPIRES].

[20] F. Denef and S.A. Hartnoll, Landscape of superconducting membranes, Phys. Rev. D 79 (2009) 126008 [arXiv:0901.1160] [SPIRES].

[21] G. Koutsoumbas, E. Papantonopoulos and G. Siopsis, Exact gravity dual of a gapless superconductor, JHEP 07 (2009) 026 [arXiv:0902.0733] [SPIRES].

[22] C.P. Herzog and S.S. Pufu, The second sound of SU(2), JHEP 04 (2009) 126 [arXiv: 0902.0409] [SPIRES].

[23] A. Karch and E. Katz, Adding flavor to AdS/CFT, JHEP 06 (2002) 043 [hep-th/0205236] [SPIRES].

[24] M. Kruczenski, D. Mateos, R.C. Myers and D.J. Winters, Meson spectroscopy in AdS/CFT with flavour, JHEP 07 (2003) 049 [hep-th/0304032] [SPIRES].

[25] J. Babington, J. Erdmenger, N.J. Evans, Z. Guralnik and I. Kirsch, Chiral symmetry breaking and pions in non-supersymmetric gauge/gravity duals, Phys. Rev. D 69 (2004) 066007 [hep-th/0306018] [SPIRES].

[26] S. Kobayashi, D. Mateos, S. Matsuura, R.C. Myers and R.M. Thomson, Holographic phase transitions at finite baryon density, JHEP 02 (2007) 016 [hep-th/0611099] [SPIRES].

[27] A. Karch and A. O'Bannon, Holographic thermodynamics at finite baryon density: some exact results, JHEP 11 (2007) 074 [arXiv:0709.0570] [SPIRES].

[28] K. Ghoroku, M. Ishihara and A. Nakamura, D3/D7 holographic gauge theory and chemical potential, Phys. Rev. D 76 (2007) 124006 [arXiv:0708.3706] [SPIRES].

[29] D. Mateos, S. Matsuura, R.C. Myers and R.M. Thomson, Holographic phase transitions at finite chemical potential, JHEP 11 (2007) 085 [arXiv:0709.1225] [SPIRES]. 
[30] S. Nakamura, Y. Seo, S.-J. Sin and K.P. Yogendran, A new phase at finite quark density from AdS/CFT, J. Korean Phys. Soc. 52 (2008) 1734 [hep-th/0611021] [SPIRES].

[31] S. Nakamura, Y. Seo, S.-J. Sin and K.P. Yogendran, Baryon-charge chemical potential in AdS/CFT, Prog. Theor. Phys. 120 (2008) 51 [arXiv:0708.2818] [SPIRES].

[32] J. Erdmenger, M. Kaminski, P. Kerner and F. Rust, Finite baryon and isospin chemical potential in AdS/CFT with flavor, JHEP 11 (2008) 031 [arXiv:0807.2663] [SPIRES].

[33] J. Erdmenger, M. Kaminski and F. Rust, Holographic vector mesons from spectral functions at finite baryon or isospin density, Phys. Rev. D 77 (2008) 046005 [arXiv:0710.0334] [SPIRES].

[34] R. Apreda, J. Erdmenger, N. Evans and Z. Guralnik, Strong coupling effective Higgs potential and a first order thermal phase transition from AdS/CFT duality, Phys. Rev. D 71 (2005) 126002 [hep-th/0504151] [SPIRES].

[35] A. Buchel, J. Jia and V.A. Miransky, Dynamical stabilization of runaway potentials at finite density, Phys. Lett. B 647 (2007) 305 [hep-th/0609031] [SPIRES].

[36] O. Aharony, K. Peeters, J. Sonnenschein and M. Zamaklar, Rho meson condensation at finite isospin chemical potential in a holographic model for QCD, JHEP 02 (2008) 071 [arXiv: 0709.3948] [SPIRES].

[37] G.E. Brown and M. Rho, Scaling effective lagrangians in a dense medium, Phys. Rev. Lett. 66 (1991) 2720 [SPIRES].

[38] F. Sannino, General structure of relativistic vector condensation, Phys. Rev. D 67 (2003) 054006 [hep-ph/0211367] [SPIRES].

[39] D. Mateos and L. Patino, Bright branes for strongly coupled plasmas, JHEP 11 (2007) 025 [arXiv:0709.2168] [SPIRES].

[40] D.T. Son and M.A. Stephanov, QCD at finite isospin density, Phys. Rev. Lett. 86 (2001) 592 [hep-ph/0005225] [SPIRES].

[41] D.T. Son and M.A. Stephanov, QCD at finite isospin density: from pion to quark antiquark condensation, Phys. Atom. Nucl. 64 (2001) 834 [hep-ph/0011365] [SPIRES].

[42] J.B. Kogut and D.K. Sinclair, Lattice QCD at finite isospin density at zero and finite temperature, Phys. Rev. D 66 (2002) 034505 [hep-lat/0202028] [SPIRES].

[43] J. Erdmenger, N. Evans, I. Kirsch and E. Threlfall, Mesons in gauge/gravity duals - A review, Eur. Phys. J. A 35 (2008) 81 [arXiv:0711.4467] [SPIRES].

[44] R.C. Myers, Dielectric-branes, JHEP 12 (1999) 022 [hep-th/9910053] [SPIRES].

[45] A.A. Tseytlin, On non-abelian generalisation of the Born-Infeld action in string theory, Nucl. Phys. B 501 (1997) 41 [hep-th/9701125] [SPIRES].

[46] A. Hashimoto and W. Taylor, Fluctuation spectra of tilted and intersecting D-branes from the Born-Infeld action, Nucl. Phys. B 503 (1997) 193 [hep-th/9703217] [SPIRES].

[47] N.R. Constable, R.C. Myers and O. Tafjord, The noncommutative bion core, Phys. Rev. D 61 (2000) 106009 [hep-th/9911136] [SPIRES].

[48] R.C. Myers and M.C. Wapler, Transport properties of holographic defects, JHEP 12 (2008) 115 [arXiv:0811.0480] [SPIRES].

[49] A. Karch, A. O'Bannon and K. Skenderis, Holographic renormalization of probe D-branes in AdS/CFT, JHEP 04 (2006) 015 [hep-th/0512125] [SPIRES]. 
[50] D. Mateos, R.C. Myers and R.M. Thomson, Thermodynamics of the brane, JHEP 05 (2007) 067 [hep-th/0701132] [SPIRES].

[51] A. Karch, D.T. Son and A.O. Starinets, Zero sound from holography, arXiv:0806.3796 [SPIRES].

[52] T. Faulkner and H. Liu, Condensed matter physics of a strongly coupled gauge theory with quarks: some novel features of the phase diagram, arXiv:0812.4278 [SPIRES].

[53] C. Hoyos-Badajoz, K. Landsteiner and S. Montero, Holographic meson melting, JHEP 04 (2007) 031 [hep-th/0612169] [SPIRES].

[54] D.T. Son and A.O. Starinets, Minkowski-space correlators in AdS/CFT correspondence: recipe and applications, JHEP 09 (2002) 042 [hep-th/0205051] [SPIRES].

[55] M. Tinkham, The electromagnetic properties of superconductors, Rev. Mod. Phys. 46 (1974) 587 [SPIRES].

[56] A. Karch and A. O'Bannon, Metallic AdS/CFT, JHEP 09 (2007) 024 [arXiv:0705.3870] [SPIRES].

[57] A. Karch, A. O'Bannon and E. Thompson, The stress-energy tensor of flavor fields from AdS/CFT, JHEP 04 (2009) 021 [arXiv:0812.3629] [SPIRES].

[58] R.C. Myers, A.O. Starinets and R.M. Thomson, Holographic spectral functions and diffusion constants for fundamental matter, JHEP 11 (2007) 091 [arXiv:0706.0162] [SPIRES]. 